\title{
Long-range fermions and critical dualities
}

\author{
Noam Chai, ${ }^{a}$ Soumangsu Chakraborty, ${ }^{b}$ Mikhail Goykhman $^{c}$ and Ritam Sinha ${ }^{a}$ \\ ${ }^{a}$ The Racah Institute of Physics, The Hebrew University of Jerusalem, \\ Jerusalem 91904, Israel \\ ${ }^{b}$ Department of Theoretical Physics, Tata Institute of Fundamental Research, \\ omi Bhabha Road, Mumbai 400005, India \\ ${ }^{c}$ William I. Fine Theoretical Physics Institute, University of Minnesota, \\ Minneapolis, MN 55455, U.S.A. \\ E-mail: ritam.sinha@mail.huji.ac.il, goykhman@umn.edu, \\ soumangsuchakraborty@gmail.com, ritam.sinha@mail.huji.ac.il
}

ABSTRACT: We construct long-range fermionic models with the Gross-Neveu and GrossNeveu-Yukawa interaction, and argue that their critical regimes are equivalent. To this end, we calculate various CFT data in $\epsilon$ - and $1 / N$ - expansion, and demonstrate their agreement in the overlapping regimes of validity.

Keywords: $1 / N$ Expansion, Conformal Field Theory, Global Symmetries, Nonperturbative Effects

ARXIV EPRINT: 2110.00020 


\section{Contents}

1 Introduction 1

2 Brief review of short-range Gross-Neveu model 4

3 Long-range Gross-Neveu model $\quad 5$

$3.1\langle\psi \bar{\psi}\rangle \quad 8$

$3.2\langle\sigma \sigma\rangle \quad 9$

$3.3 \sigma \bar{\psi} \psi$ conformal triangle $\quad 11$

$\begin{array}{lll}3.4\langle\sigma \bar{\psi} \psi\rangle & 13\end{array}$

$\begin{array}{ll}3.5 \text { Continuity of CFT data at } s=s_{\star} & 15\end{array}$

4 Long-range Gross-Neveu-Yukawa model $\quad 15$

$\begin{array}{lll}4.1\langle\phi\rangle & 18\end{array}$

$\begin{array}{ll}4.2\langle\phi \bar{\theta} \theta\rangle & 19\end{array}$

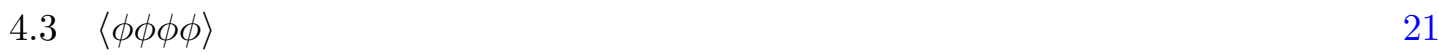

5 Discussion $\quad 23$

$\begin{array}{ll}\text { A Some useful identities } & 24\end{array}$

\section{Introduction}

The Gross-Neveu (GN) model describes dynamics of fermions in fundamental representation of the $\mathrm{U}(n)$ symmetry group, coupled via the four-fermionic interaction term [1]. In two dimensions, this model provides a toy example of quantum chromodynamics, featuring such properties as asymptotic freedom, dynamical chiral symmetry breaking, and mass gap generation via dimensional transmutation. In $2<d<4$ dimensions the four-fermionic interaction is power-counting non-renormalizable. However, the Gross-Neveu model is renormalizable when expanded in $1 / n$ for a large number $n$ of fermionic flavors $[1,2]$.

In fact, for $2<d<4$, renormalization group ( $R G$ ) flow of the four-fermionic coupling constant brings the model to a fixed point in the ultra-violet (UV) limit, manifesting a non-trivial interacting critical regime at high energies. Critical regimes at fixed points of RG flows can be studied via techniques of conformal field theory (CFT) [3]. ${ }^{1}$ The same

\footnotetext{
${ }^{1}$ In a weakly-coupled regime, one can explicitly solve for the fixed point values of coupling constants using perturbative $\epsilon$-expansion [4]. In a general non-perturbative regime, but at large $N$, existence of a nontrivial CFT at the end of an RG flow in vector models can be argued for using the Hubbard-Stratonovich transformation [5]. By demanding that the Hubbard-Stratonovich field obeys the unitarity bound, one constrains the allowed space-time dimension [6]. In case of the Gross-Neveu model, this leads to the requirement $d<4[1,2]$.
} 
critical regime has been argued to be attained at the infra-red (IR) end of an RG flow of a different system, the Gross-Neveu-Yukawa (GNY) model [2]. Since one of these models achieves its interacting critical regime in the UV, while another one is taken in the IR, one can in fact view this as a UV completion.

A bosonic model counterpart of such a UV completion has recently been argued to exist for the $O(N)$ vector model with quartic interaction [7]. When considered in higher dimensions, $4<d<6$, the quartic scalar coupling is power-counting non-renormalizable. However, critical regime of the large- $N$ version of this model, achieved in the UV, can be explored in the $1 / N$ expansion [6]. A UV completion of this model was proposed in [7], where it was argued that the same CFT can be found at the IR end of an RG flow of the $O(N)$ vector model coupled to a fundamental singlet scalar field with a cubic selfinteraction. When considered in overlapping regimes of validity, the corresponding CFT data were shown to match for these two models [7-9].

A different class of critical models is given by spin systems with long-range interaction, and their continuous version, provided by generalized free scalar field perturbed by a local quartic interaction term [10-13]. These models are characterized by an extra parameter $s$, controlling the exponent of the power-law decay of the long-range bi-local kinetic term of the generalized free field. When $s>d / 2$, the quartic coupling constant is relevant, and these models reach a non-trivial critical regime in the IR (when $s<d / 2$, the IR regime is described by the mean-field theory). The $O(N)$ vector model version of these models has been explored in [14-16].

For $s$ greater than the crossover value, $s>s_{\star}=2-2 \gamma_{\hat{\phi}}$, where $\gamma_{\hat{\phi}}$ is anomalous dimension of the short-range fundamental scalar field $\hat{\phi}$, the model smoothly transitions to the short-range critical regime $[17,18]$. The nature of this short-range model has been recently elucidated in $[19,20]$. It is given by the critical short-range model with quartic self-interaction plus a decoupled generalized free field $\chi$ with scaling dimension $(d+s) / 2$.

On the other hand, in the long-range domain $d / 2<s<s_{\star}, 1<d<4$, one obtains a rich critical IR behavior, controlled by the parameter $s$. While long-range models do not possess a stress-energy tensor, substantial evidence has been accumulated that this interacting critical regime is in fact described by a CFT [19-22] (see also [16] for the investigation of conformal symmetry in the large- $N$ long-range $O(N)$ vector model, and [14, 15, 23-27] for previous calculations of critical exponents). Moreover, the same CFT was argued in $[19,20]$ to be the IR end-point of an RG flow triggered by coupling the shortrange vector field $\hat{\phi}$ to the generalized free field $\chi$ of dimension $(d+s) / 2$ via the bi-linear term $\lambda \int d^{d} x \hat{\phi} \chi$. When $s>s_{\star}$, the coupling $\lambda$ is irrelevant, and the field $\chi$ decouples. For $s$ slightly below $s_{\star}$, the IR fixed point $\lambda_{\star}$ can be studied perturbatively. $O(N)$ generalization of this duality has recently been discussed in [16].

In the regime of $s<d / 2$, the quartic interaction of the long-range vector model becomes irrelevant. Therefore the IR regime of the model is described by mean-field theory. However, RG flow of the quartic coupling drives the theory to a non-trivial regime in the UV. The corresponding CFT can be studied using the $1 / N$ expansion. In fact, the results of [14-16] for various anomalous dimensions and OPE coefficients, derived for general $d$ 
and $s$, can be formally continued to this regime. It was argued in [28] that for $s<d / 2$ the long-range $O(N)$ vector model achieves a non-trivial critical regime in the UV. This situation is analogous to a non-trivial UV criticality of the usual local (short-range) $O(N)$ vector model in $4<d<6$. Having this analogy in mind, [28] proposed a long-range version of the cubic model of [7] and provided perturbative evidence that the IR stable fixed point of this cubic model is equivalent to the UV fixed point of the long-range $O(N)$ vector model for $d / 3<s<\min \left(d / 2, s_{\star}\right), 1<d<6$.

In this paper, we are interested in constructing long-range version of the Gross-Neveu model. We will begin by formulating a generalized free field theory action for the Dirac fermion multiplet $\psi^{i}, i=1, \ldots, n$ in fundamental representation of the $\mathrm{U}(n)$ symmetry group. Power-law decay of the long-range interaction will be controlled by an exponent parameter $s$. We will then perturb this model by the local four-fermionic self-interaction of the Gross-Neveu type, and argue that the resulting system flows to an interacting regime in the UV in the domain ${ }^{2}$

$$
d / 2<s<\min \left(d / 2+1, s_{\star}\right), \quad 1<d<4 .
$$

Here $s_{\star}=2-2 \gamma_{\hat{\psi}}$ is the crossover value of the exponent $s$, above which the model transitions to the critical short-range regime, involving dynamics of the interacting fermionic multiplet $\hat{\psi}^{i}, i=1, \ldots, n$ with the anomalous dimension $\gamma_{\hat{\psi}}$.

Working within the Hubbard-Stratonovich framework, we will calculate various CFT data $^{3}$ in the long-range Gross-Neveu model, as a function of general $d, s$, at the next-toleading order in $1 / N$ expansion. In particular, we are interested in anomalous dimension of the Hubbard-Stratonovich field, $\sigma$, and the OPE coefficient $\langle\sigma \bar{\psi} \psi\rangle$. We will explicitly verify that all of our CFT data is in fact continuous at the long-range-short-range crossover point $s_{\star}$.

As we have reviewed above, the short-range Gross-Neveu-Yukawa model furnishes a UV completion of the short-range Gross-Neveu model [2]. Motivated by this, and encouraged by the recent example [28], we proceed to constructing the long-range version of the GrossNeveu-Yukawa model. Working within the scope of perturbative $\epsilon$-expansion, we derive the IR stable fixed point of this model, and calculate various CFT data at the fixed point. Assembling all the results together, and expanding them in overlapping regimes of validity, we explicitly demonstrate critical equivalence of the long-range Gross-Neveu and GrossNeveu-Yukawa models. We then suggest that the critical duality in fact holds in the entire domain (1.1).

The rest of this paper is organized as follows. In section 2 we collect some known CFT data in the short-range Gross-Neveu model. In section 3 we construct the long-range Gross-

\footnotetext{
${ }^{2}$ See also [29] for a similar model involving long-range fermions.

${ }^{3}$ Recall that while the long-range vector models are well-defined in the Euclidean signature, their naive continuation to the Lorentzian signature can result in the lack of causality. These issues are beyond the scope of the present paper. Instead, in the subsequent discussion we always work in the Euclidean signature. At the same time, it is important to remark that since the long-range models do not possess a local stressenergy tensor, it is not true a priori that correlation functions are conformally covariant. Accordingly, all the specific correlation functions that we calculate in this paper are derived without invoking any assumptions of conformal symmetry.
} 
Neveu model, and establish the parameter domain (1.1) in which it flows to an interacting critical regime in the UV. Working at the next-to-leading order in the $1 / N$ expansion, we derive various CFT data. We also explicitly demonstrate continuity of CFT data at the long-range-short-range crossover point $s_{\star}$, using various expressions for the short-range model, collected in section 2. In section 4 we construct the long-range Gross-Neveu-Yukawa model. Working perturbatively at the next-to-leading order in couplings, we find the IR stable fixed point of this model, and calculate anomalous dimension of the scalar field at the fixed point. We perform comparison of various CFT data in the long-range Gross-Neveu and Gross-Neveu-Yukawa models in the overlapping regimes of validity, and demonstrate their agreement. We discuss our results in section 5. Some known identities, useful for CFT calculations in position space, are collected in appendix A.

\section{Brief review of short-range Gross-Neveu model}

As we discussed in section 1, we expect to observe a smooth crossover between the longrange and the short-range critical $\mathrm{U}(n)$ fermionic models with the Gross-Neveu interaction at the threshold value $s_{\star}$ of the long-range exponent parameter $s$. This threshold value can be expressed in terms of anomalous dimension of the short-range fermion $\hat{\psi}$ as $s_{\star}=2-2 \gamma_{\hat{\psi}}$. In the large- $N$ limit, that we are going to be mostly interested in when studying GrossNeveu interaction, the fermion anomalous dimension is given by $[2,30]$

$$
\gamma_{\hat{\psi}}=-\frac{1}{N} \frac{2^{d-1} \sin \left(\frac{\pi d}{2}\right) \Gamma\left(\frac{d-1}{2}\right)}{\pi^{3 / 2} d \Gamma\left(\frac{d}{2}-1\right)}+\mathcal{O}\left(\frac{1}{N^{2}}\right) .
$$

To facilitate test of smooth CFT crossover, in this section we collect some known CFT data in the short-range Gross-Neveu model $[1]^{4}$

$$
\left.S_{\mathrm{SR} \mathrm{GN}}=\int d^{d} x\left(\overline{\hat{\psi}}^{i} \gamma^{\mu} \partial_{\mu} \hat{\psi}^{i}+\frac{\hat{g}}{N}(\overline{\hat{\psi}} \hat{\psi})^{2}\right)\right)
$$

where we a sum over repeated $\mathrm{U}(n)$ index $i=1, \ldots, n$ is implied. Performing the HubbardStratonovich transformation, we can re-write (2.2) as (we skip U( $n)$ indices in what follows for the sake of brevity)

$$
S_{\mathrm{SR} \mathrm{GN}}=\int d^{d} x\left(\overline{\hat{\psi}} \gamma^{\mu} \partial_{\mu} \hat{\psi}-\frac{1}{4 \hat{g}} \hat{\sigma}^{2}+\frac{1}{\sqrt{N}} \hat{\sigma} \overline{\hat{\psi}} \hat{\psi}\right) .
$$

While at the free fixed point, $\hat{g}=0$, scaling dimension of the pseudo-scalar HubbardStratonovich field $\hat{\sigma} \sim \hat{\bar{\psi}} \hat{\psi}$ is given by $[\hat{\sigma}]_{\text {free }}=d-1$, at the interacting fixed point, achieved in the UV for $2<d<4$, it is given by $[\hat{\sigma}]_{\mathrm{UV}}=1+\gamma_{\hat{\sigma}}$, where $[2]$

$$
\gamma_{\hat{\sigma}}=\frac{1}{N} \frac{4 \sin \left(\frac{\pi d}{2}\right) \Gamma(d)}{\pi d \Gamma\left(\frac{d}{2}\right)^{2}}+\mathcal{O}\left(\frac{1}{N^{2}}\right) .
$$

\footnotetext{
${ }^{4}$ Euclidean gamma-matrices are Hermitian, $\left(\gamma^{\mu}\right)^{\dagger}=\gamma^{\mu}$, satisfying $\gamma^{\mu} \gamma^{\nu}+\gamma^{\nu} \gamma^{\mu}=2 \delta^{\mu \nu} \mathbb{I}$, where $\mathbb{I}$ is rank-2 ${ }^{[d / 2]}$ unit matrix. Dirac conjugate of a spinor is an ordinary Hermitian conjugate, $\bar{\psi}=\psi^{\dagger}$. Following the standard conventions in large- $N$ GN model [2], we defined $N=2^{[d / 2]} n$.
} 
Besides the scaling dimensions (2.1), (2.4), another non-trivial piece of CFT data is given by the amplitude of the three-point function $[31]^{5}$

$$
\begin{aligned}
\left.\left\langle\overline{\hat{\psi}}\left(x_{1}\right) \hat{\psi}\left(x_{2}\right) \hat{\sigma}\left(x_{3}\right)\right\rangle\right|_{\text {normalized }} & =C_{\overline{\hat{\psi}} \hat{\psi} \hat{\sigma}}\left(1+\delta C_{\overline{\hat{\psi}} \hat{\psi} \hat{\sigma}}\right) \frac{\gamma_{\mu} x_{13}^{\mu} \gamma_{\nu} x_{32}^{\nu}}{\left|x_{12}\right|^{d-2}\left|x_{13}\right|^{2}\left|x_{23}\right|^{2}} \\
C_{\overline{\hat{\psi} \hat{\psi} \hat{\sigma}}} & =-\frac{2^{\frac{d}{2}}}{\sqrt{N}(d-2) \pi^{\frac{3}{4}}} \sqrt{-\frac{\sin \left(\frac{\pi d}{2}\right) \Gamma\left(\frac{d-1}{2}\right)}{\Gamma\left(\frac{d}{2}-1\right)}} \\
\delta C_{\overline{\hat{\psi} \hat{\psi} \hat{\sigma}}} & =\frac{2(d-1)\left((d-2) H_{d-2}+\pi(d-2) \cot \left(\frac{\pi d}{2}\right)-2\right)}{(d-2)^{2}} \gamma_{\hat{\psi}}
\end{aligned}
$$

where propagators of all fields in position space have been normlalized to unity, and $H_{n}$ is the $n$th Harmonic number.

\section{Long-range Gross-Neveu model}

Consider generalized (long-range) free Dirac fermion multiplet in fundamental representation of $\mathrm{U}(n)$, defined in $d$-dimensional Euclidean space, and described by the bi-local action

$$
S_{\mathrm{LR} \operatorname{Dirac}}[\psi]=C_{f}(s) \int d^{d} x \int d^{d} y \bar{\psi}^{i}(x) \gamma^{\mu} \psi^{i}(y) \frac{(x-y)^{\mu}}{|x-y|^{d+s}},
$$

where sum over repeated $i=1, \ldots, n$ is implied. Here $s$ is a free parameter, that determines the fermion scaling dimension

$$
\Delta_{\psi}=\frac{d-s+1}{2} .
$$

We choose the pre-factor $C_{f}(s)$ so that the free fermionic propagator is canonically normalized in momentum space,

$$
\langle\psi(k) \bar{\psi}(p)\rangle=(2 \pi)^{d} \delta^{(d)}(p+q) \frac{-i \gamma^{\mu} k_{\mu}}{\left(k^{2}\right)^{s / 2}} .
$$

This gives ${ }^{6}$

$$
C_{f}(s)=-\frac{2^{s-1} \Gamma\left(\frac{d+s}{2}\right)}{\pi^{\frac{d}{2}} \Gamma\left(1-\frac{s}{2}\right)} .
$$

When $s=2$, we recover the usual (short-range) Dirac fermion propagator, while the unitarity bound imposes the constraint

$$
s \leq 2
$$

\footnotetext{
${ }^{5}$ See also [32] for earlier derivation of the $\hat{\sigma} \hat{\bar{\psi}} \hat{\psi}$ conformal triangle and $1 / N$ corrections to the $\langle\hat{\sigma} \hat{\sigma}\rangle$ propagator.

${ }^{6}$ One can verify this expression using the Fourier transform

$$
\int \frac{d^{d} k}{(2 \pi)^{d}} e^{i k \cdot x} \frac{1}{\left(k^{2}\right)^{\frac{d}{2}-\Delta}}=\frac{2^{2 \Delta-d}}{\pi^{\frac{d}{2}}} \frac{\Gamma(\Delta)}{\Gamma\left(\frac{d}{2}-\Delta\right)} \frac{1}{|x|^{2 \Delta}} .
$$
}


In position space, for general $s$, we obtain

$$
\langle\psi(x) \bar{\psi}(0)\rangle=C_{\psi} \frac{\gamma^{\mu} x_{\mu}}{|x|^{2 \Delta_{\psi}+1}},
$$

where $\Delta_{\psi}$ is defined by (3.2), and the free propagator amplitude is given by

$$
C_{\psi}=\frac{\Gamma\left(\frac{d-s}{2}+1\right)}{2^{s-1} \pi^{\frac{d}{2}} \Gamma\left(\frac{s}{2}\right)} .
$$

Notice that when $s \rightarrow 2$ we recover the corresponding quantities of the free short-range Dirac fermion ${ }^{7}$

$$
\left.\Delta_{\psi}\right|_{s \rightarrow 2}=\Delta_{\hat{\psi}},\left.\quad C_{\psi}\right|_{s \rightarrow 2}=C_{\hat{\psi}}
$$

Let us now deform the action (3.1) by a quartic (Gross-Neveu) fermion selfinteraction term, ${ }^{8}$

$$
S_{\mathrm{LR} \mathrm{GN}}=S_{\mathrm{LR} \text { Dirac }}+\frac{g}{N} \int d^{d} x(\bar{\psi} \psi)^{2} .
$$

In the vicinity of the free regime (3.1) we obtain $[g]=2 s-2-d$. Therefore the quartic interaction in (3.9) is a relevant perturbation when $s>d / 2+1$. Together with the unitarity constraint (3.5), this imposes $d<2$. In this case, the GN interaction will drive the theory to a non-trivial regime in the IR. For $s=d / 2+1+\epsilon$ the interaction strength is weak, and one can perform a Wilson-Fisher perturbative $\epsilon$-expansion [4].

Instead, in this paper we are going to focus on the domain where $s<d / 2+1$, that results in an irrelevant GN interaction term, driving the theory to an interacting regime in the UV. Together with the unitarity constraint (3.5), we obtain

$$
s<\min \left(\frac{d}{2}+1,2\right) .
$$

Therefore, when $d<2$, we achieve a non-trivial regime in the UV for $s<d / 2+1$ and in the IR for $s>d / 2+1$. For $d>2$, we can only achieve a non-trivial regime in the UV, for $s<2$.

Analogously to the bosonic case [17, 18], the long-range model crosses over to the short-range regime for $s>s_{\star}$, where the threshold value of the exponent parameter is given by

$$
s_{\star}=2-2 \gamma_{\hat{\psi}} .
$$

Due to $\gamma_{\hat{\psi}}>0$, we obtain from (3.10)

$$
s<\min \left(\frac{d}{2}+1, s_{\star}\right) .
$$

To perform large- $N$ analysis of the long-range GN model (3.9), it is convenient to introduce the Hubbard-Stratonovich pseudo-scalar field $\sigma \sim \bar{\psi} \psi$, and rewrite the action as

$$
S_{\mathrm{LR} \mathrm{GN}}=S_{\mathrm{LR} \text { Dirac }}+\int d^{d} x\left(-\frac{1}{4 g} \sigma^{2}+\frac{1}{\sqrt{N}} \sigma \bar{\psi} \psi\right)
$$

\footnotetext{
${ }^{7}$ See [31] for a recent review.

${ }^{8}$ When $s=1$, the model can be seen as a free fermion in $d+1$-dimensional bulk, with the interaction localized on the boundary. See also [33] for discussion of dualities between fixed points of free vector models with interactions localized on the boundary.
} 
Importantly, the model (3.13) enjoys the discrete $\mathbb{Z}_{2}$ symmetry,

$$
\begin{aligned}
\left(x^{1}, \ldots, x^{a-1}, x^{a}, x^{a+1}, \ldots, x^{d}\right) & \rightarrow\left(x^{1}, \ldots, x^{a-1},-x^{a}, x^{a+1}, \ldots, x^{d}\right), \\
\sigma \rightarrow-\sigma, \quad \psi & \rightarrow \gamma^{a} \psi, \quad \bar{\psi} \rightarrow-\bar{\psi} \gamma^{a},
\end{aligned}
$$

defined for any given $a=1, \ldots, d$. This is analogous to the local (short-range) Gross-Neveu model (2.3), that possesses the same symmetry [1, 2, 34].

Integrating out the fermions, we arrive at the effective action for the HubbardStratonovich field:

$$
\begin{aligned}
S_{\mathrm{LR} \mathrm{GN}}= & -n \int d^{d} x \int d^{d} y \operatorname{tr} \log \left(C_{f}(s) \frac{(x-y)_{\mu} \gamma^{\mu}}{|x-y|^{d+s}}+\frac{1}{\sqrt{N}} \sigma(x) \delta^{(d)}(x-y)\right) \\
& -\frac{1}{4 g} \int d^{d} x \sigma^{2},
\end{aligned}
$$

where trace is taken over gamma-matrices. Expanding the logarithm in (3.15) to leading order in $1 / N$, while discarding the constant and tadpole terms, we obtain ${ }^{9}$

$$
S_{\mathrm{LR} \mathrm{GN}}=\frac{C_{\psi}^{2}}{2} \int d^{d} x \int d^{d} y \frac{\sigma(x) \sigma(y)}{|x-y|^{2(d-s+1)}}-\frac{1}{4 g} \int d^{d} x \sigma^{2}+\mathcal{O}\left(\frac{1}{N}\right) .
$$

At the interacting fixed point in the UV, the first term in (3.16) dominates over the second term. Indeed, the corresponding propagator for the Hubbard-Stratonovich field (at the leading order in $1 / N$ ) is given by

$$
\langle\sigma(x) \sigma(0)\rangle=\frac{C_{\sigma}}{|x|^{2 \Delta_{\sigma}}},
$$

where we defined the free propagator amplitude

$$
C_{\sigma}=-\frac{4^{s-1} \Gamma(s-1) \Gamma\left(\frac{s}{2}\right)^{2} \Gamma(d-s+1)}{\Gamma\left(\frac{d}{2}-s+1\right) \Gamma\left(\frac{d-s}{2}+1\right)^{2} \Gamma\left(-\frac{d}{2}+s-1\right)},
$$

and scaling dimension

$$
\Delta_{\sigma}=s-1
$$

In other words, at the interacting UV f.p. we obtain

$$
[\sigma]_{\mathrm{UV}}=s-1+\mathcal{O}\left(\frac{1}{N}\right) .
$$

We then obtain $\left[\sigma^{2}\right]_{\mathrm{UV}}=2(s-1)+\mathcal{O}\left(\frac{1}{N}\right)<d$. Therefore the second term in (3.16) is irrelevant in the UV. As a consistency check, in the $s \rightarrow 2$ limit we can reproduce the corresponding quantities at the UV fixed point of the local (short-range) GN model [2, 32, 35],

$$
\left.\Delta_{\sigma}\right|_{s \rightarrow 2}=\Delta_{\hat{\sigma}},\left.\quad C_{\sigma}\right|_{s \rightarrow 2}=C_{\hat{\sigma}} .
$$

\footnotetext{
${ }^{9}$ Here we use the free fermionic inverse propagator relation:

$$
\int d^{d} z \frac{C_{f}(s)(x-z)^{\mu} \gamma_{\mu}}{|x-z|^{d+s}} \frac{C_{\psi} z^{\nu} \gamma_{\nu}}{|z|^{d-s+2}}=\delta^{(d)}(x) \mathbb{I},
$$
}

that can be verified, for instance, using the Fourier transform. 
Demanding unitarity, we impose $[\sigma]_{\mathrm{UV}}>\frac{d}{2}-1$, that in turn demands $s>d / 2$. Combing with (3.12), we obtain

$$
\frac{d}{2}<s<\min \left(\frac{d}{2}+1, s_{\star}\right)
$$

and therefore

$$
1<d<4 \text {. }
$$

Having established propagators for the fermion and the Hubbard-Stratonovich fields, as well as the cubic interaction vertex, at the leading order in $1 / N$, we return to the formulation of the model in terms of the action (3.13). The corresponding Feynman rules that will be used at the interacting UV fixed point in diagrammatic calculations in this section are given by
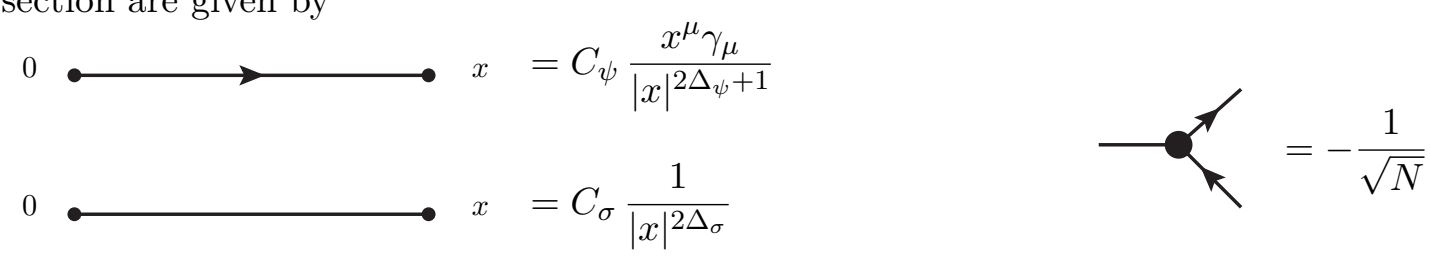

When exponents of the propagator lines are specified explicitly, the corresponding propagators will be implied to have unit amplitudes:

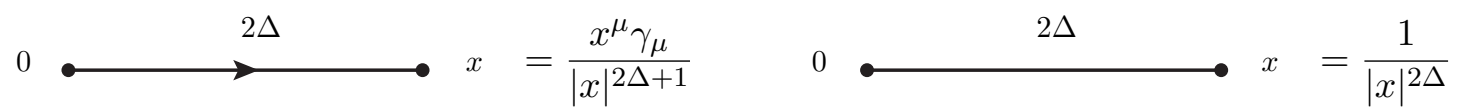

Recall also that each closed fermionic line produces the factor of -1 due to the anticommuting nature of fermions.

\section{$3.1\langle\psi \bar{\psi}\rangle$}

At the leading order in $1 / N$ expansion, the $\langle\psi \bar{\psi}\rangle$ propagator is given by the generalized free field theory expression (3.6). The scaling dimension $\Delta_{\psi}$, given by (3.2), is protected from loop corrections by the bi-local kinetic term, and therefore the anomalous dimension of $\psi$ vanishes, $\gamma_{\psi}=0$, to all orders in $1 / N$ expansion. However, while in the free limit the amplitude of the propagator $C_{\psi}$ is given by (3.7), the $1 / N$ corrections are going to renormalize it, resulting in

$$
\langle\psi(x) \bar{\psi}(0)\rangle=C_{\psi}\left(1+A_{\psi}\right) \frac{\gamma^{\mu} x_{\mu}}{|x|^{2 \Delta_{\psi}+1}} .
$$

Our goal in this section is to find the relative correction to the propagator amplitude $A_{\psi}$ at the next-to-leading order in $1 / N$ expansion. The only contributing diagram is one-loop, given by:

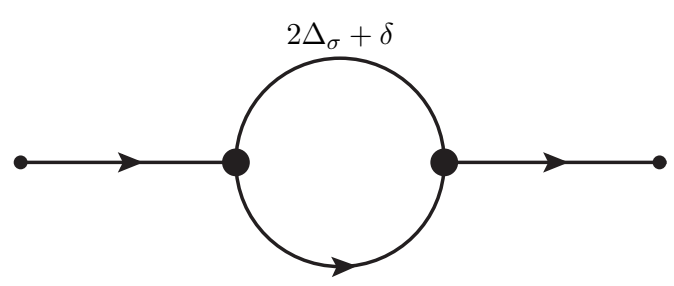


The standard technique to renormalize such a conformal graph is to add a small shift $\delta$ to the exponents of the internal $\sigma$ lines [36]. Subsequently using the loop identities in position space, as well as the propagator merging relations, reviewed in appendix A, we obtain

$$
\begin{aligned}
\langle\psi(x) \bar{\psi}(0)\rangle \supset & -\frac{1}{N} C_{\psi}^{3} C_{\sigma} \pi^{d} A\left(-\frac{\delta}{2}\right) V\left(\frac{d+s-1+\delta}{2}, \frac{d-s+1}{2}\right) \\
& \times A\left(\frac{d+\delta}{2}\right) V\left(\frac{d-s+1}{2}, \frac{s-1-\delta}{2}\right) \frac{x^{\mu} \gamma_{\mu}}{|x|^{d-s+2+\delta}} .
\end{aligned}
$$

This expression is in fact finite in the $\delta \rightarrow 0$ limit, in agreement with our expectation that $\gamma_{\psi}=0$. Taking this limit renders

$$
A_{\psi}=\frac{1}{N} \frac{2^{d-s-1}(d-2 s+2)}{\sqrt{\pi} \Gamma\left(\frac{d+s}{2}\right) \sin \left(\frac{\pi s}{2}\right)} \sin \left(\frac{\pi(d-2 s)}{2}\right) \Gamma(s-1) \Gamma\left(\frac{d-s+1}{2}\right) .
$$

While propagator amplitudes are not a part of universal CFT data, in combination with the other non-observable, given by the amplitude of the $\sigma \bar{\psi} \psi$ conformal triangle, discussed in section 3.3, the expression (3.26) will play an important role in calculation of the OPE coefficient $\langle\sigma \bar{\psi} \psi\rangle$, that we are going to carry out in section 3.4.

\section{$3.2\langle\sigma \sigma\rangle$}

At the interacting UV fixed point, dimension of the Hubbard-Stratonovich field, $\sigma \simeq \bar{\psi} \psi$, is given by (3.20), where the leading order in $1 / N$ contribution is given by (3.19). When $1 / N$ corrections are taken into account, the leading order $\langle\sigma \sigma\rangle$ propagator (3.17) gets modified, acquiring the form

$$
\langle\sigma(x) \sigma(0)\rangle=\frac{C_{\sigma}\left(1+A_{\sigma}\right) \mu^{-2 \gamma_{\sigma}}}{|x|^{2\left(\Delta_{\sigma}+\gamma_{\sigma}\right)}},
$$

where $\mu$ is an arbitrary scale. Our goal in this section is to find anomalous dimension $\gamma_{\sigma}$ at the next-to-leading order in $1 / N$ expansion. This constitutes an important part of CFT data of the corresponding critical theory. We will also calculate the relative propagator amplitude correction $A_{\sigma}$, that will play a role in calculation of the $\langle\sigma \bar{\psi} \psi\rangle$ three-point function in section 3.4 .

The two-loop diagrams contributing to the $\langle\sigma \sigma\rangle$ propagator at the next-to-leading order in $1 / N$ expansion are given by: ${ }^{10}$

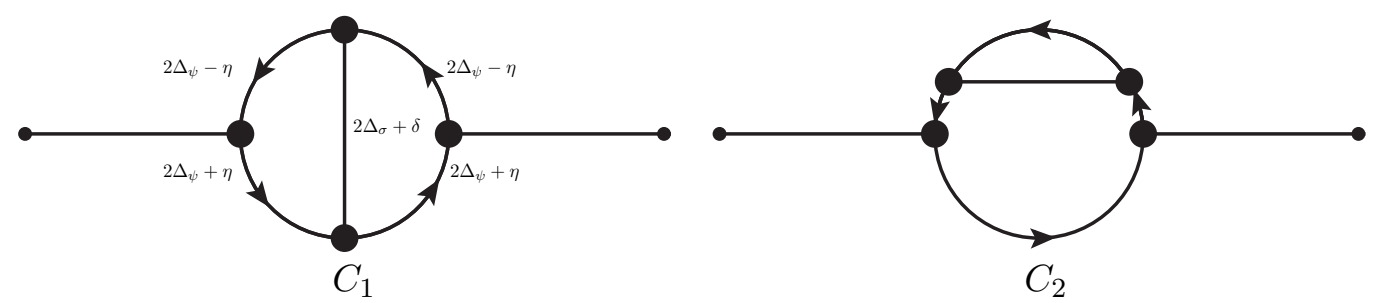

\footnotetext{
${ }^{10}$ One more $\langle\sigma \sigma\rangle$ two-loop diagram at the order $\mathcal{O}(1 / N)$ can be drawn, but it vanishes, since it contains the $\langle\sigma \sigma \sigma\rangle$ triangle sub-diagram. The latter correlation function is in fact identically zero in the Gross-Neveu model, both in the short-range [31,35], and in the long-range case, due to the $\mathbb{Z}_{2}$ symmetry (3.14). This makes some calculations in the Gross-Neveu model easier than in the bosonic $O(N)$ vector model. In the latter, vanishing of the $\langle\sigma \sigma \sigma\rangle$ three-point function is a three-dimensional artifact [37-39], and the counterpart of the $\mathbb{Z}_{2}$ symmetry does not seem to generally hold beyond the leading order in $1 / N$ expansion [40].
} 
We are going to denote the corresponding expressions contributing to $\langle\sigma \sigma\rangle$ as $C_{1,2}$. The diagram $C_{1}$ is divergent, and requires regularization that we accomplish by adding a small shift $\delta$ to the exponent of the internal $\sigma$ line [36]. Presence of $\delta$ spoils uniqueness of the three-vertices. However, using an auxiliary regulator $\eta=\delta / 2$ one can restore the integrability of the diagram, without affecting its regularized value. This is due to the fact that the diagram possesses the $\eta \rightarrow-\eta$ symmetry, ${ }^{11}$ and therefore $\eta$ dependence can only appear as $1+\mathcal{O}\left(\eta^{2}\right)$. Since the strongest divergence of this diagram is the simple pole $1 / \delta$, any choice $\eta=\mathcal{O}(\delta)$ will not influence the $\delta \rightarrow 0$ limit [14, 41-44].

Using uniqueness and propagator merging relations, reviewed in appendix A, we obtain ${ }^{12}$

$$
\begin{aligned}
C_{1}= & -\frac{1}{N} C_{\psi}^{4} C_{\sigma}^{3} \pi^{\frac{d}{2}} A\left(s-1+\frac{\delta}{2}\right) \\
& \times V\left(\frac{d-s+1}{2}-\frac{\delta}{4}, \frac{d-s+1}{2}-\frac{\delta}{4}\right) U\left(\frac{d+\delta}{2}, \frac{d+\delta}{2},-\delta\right) \\
& \times U\left(s-1, d-s+1+\frac{\delta}{2},-\frac{\delta}{2}\right) U\left(\frac{d+\delta}{2}, s-1, \frac{d-\delta}{2}-s+1\right) \frac{\mu^{-\delta}}{|x|^{2 s-2+\delta}} .
\end{aligned}
$$

Expanding around $\delta=0$, we obtain ${ }^{13}$

$$
\gamma_{\sigma}=\frac{1}{N} \frac{2 \Gamma\left(\frac{s}{2}\right)^{2} \Gamma(d-s+1)}{\Gamma\left(\frac{d}{2}\right) \Gamma\left(\frac{d-s+2}{2}\right)^{2} \Gamma\left(-\frac{d}{2}+s-1\right)}+\mathcal{O}\left(\frac{1}{N^{2}}\right) .
$$

The finite part is given by

$$
\frac{C_{1}}{C_{\sigma}} \supset \frac{1}{N} \frac{2 \Gamma\left(\frac{s}{2}\right)^{2} \Gamma(d-s+1)\left(H_{\frac{d-s}{2}}-H_{d-s}-\psi^{(0)}\left(-\frac{d}{2}+s-1\right)+\psi^{(0)}\left(\frac{s}{2}\right)\right)}{\Gamma\left(\frac{d}{2}\right) \Gamma\left(\frac{d-s}{2}+1\right)^{2} \Gamma\left(-\frac{d}{2}+s-1\right)} \equiv c_{1},
$$

where $\psi^{(n)}(x)$ is $n$th derivative of the digamma function $\psi^{(0)}(x)=\Gamma^{\prime}(x) / \Gamma(x)$.

The diagram $C_{2}$ contains the finite one-loop correction to the fermionic propagator $\langle\psi \bar{\psi}\rangle$ as a sub-diagram: ${ }^{14}$

$$
\begin{aligned}
\frac{C_{2}}{C_{\sigma}} & \supset 2 C_{\psi}^{2} C_{\sigma} A_{\psi} \pi^{d} A(s-1) A(d-s+1) \\
& =-\frac{1}{N} \frac{2^{d-s}(d-2 s+2) \Gamma(s-1) \sin \left(\frac{1}{2} \pi(d-2 s)\right) \Gamma\left(\frac{1}{2}(d-s+1)\right)}{\sqrt{\pi} \sin \left(\frac{\pi s}{2}\right) \Gamma\left(\frac{d+s}{2}\right)} \equiv c_{2},
\end{aligned}
$$

\footnotetext{
${ }^{11}$ This can be seen by renaming positions of vertices of integration, $x_{3,4} \rightarrow x_{1}+x_{2}-x_{4,3}$, where $x_{1,2}$ are left and right vertices, and $x_{3,4}$ are top and bottom vertices.

${ }^{12}$ Here the overall factor of -1 is due to the Feynman rule for the fermionic loop.

${ }^{13}$ Simple pole divergence can be removed by the wave-function renormalization $\sigma \rightarrow \sqrt{1+\frac{2 \gamma_{\sigma}}{\delta}} \sigma$. For brevity, and where it does not create confusion, we keep subtractions of pure infinities implicit in this paper.

${ }^{14}$ Here we took into account the symmetry factor of 2 , while the factor of -1 due to the fermionic loop was offset by the factor of -1 due to the simplification of loop in position space, reviewed in appendix A. We have then used the inverse propagator relation
}

$$
\int d^{d} y \frac{1}{|y|^{2 a}|x-y|^{2(d-a)}}=\pi^{d} A(a) A(d-a) .
$$


and therefore does not contribute to the anomalous dimension $\gamma_{\sigma}$. Combining (3.30), (3.31), we obtain

$$
A_{\sigma}=c_{1}+c_{2} .
$$

We close this section by providing an expansion of the anomalous dimension $\gamma_{\sigma}$, given by (3.29), at $d=4-\epsilon_{2}, s=2-\epsilon_{2} / 2+\epsilon_{1}$ to leading order in $\epsilon_{1,2}$,

$$
\left.\gamma_{\sigma}\right|_{d=4}=-\frac{4}{N} \epsilon_{1}+\mathcal{O}\left(\epsilon_{1,2}^{2}, \frac{1}{N^{2}}\right)
$$

This expansion will prove useful when we compare the CFT data of the long-range GrossNeveu model with its counterpart in the Gross-Neveu-Yukawa model.

\section{$3.3 \sigma \bar{\psi} \psi$ conformal triangle}

While the leading order $\sigma \bar{\psi} \psi$ vertex in the action (3.13) is local, higher-order $1 / N$ corrections result in a non-local effective vertex for the cubic $\sigma \bar{\psi} \psi$ interaction. Diagrammatically, in a CFT, it can be represented using the conformal triangle [3]

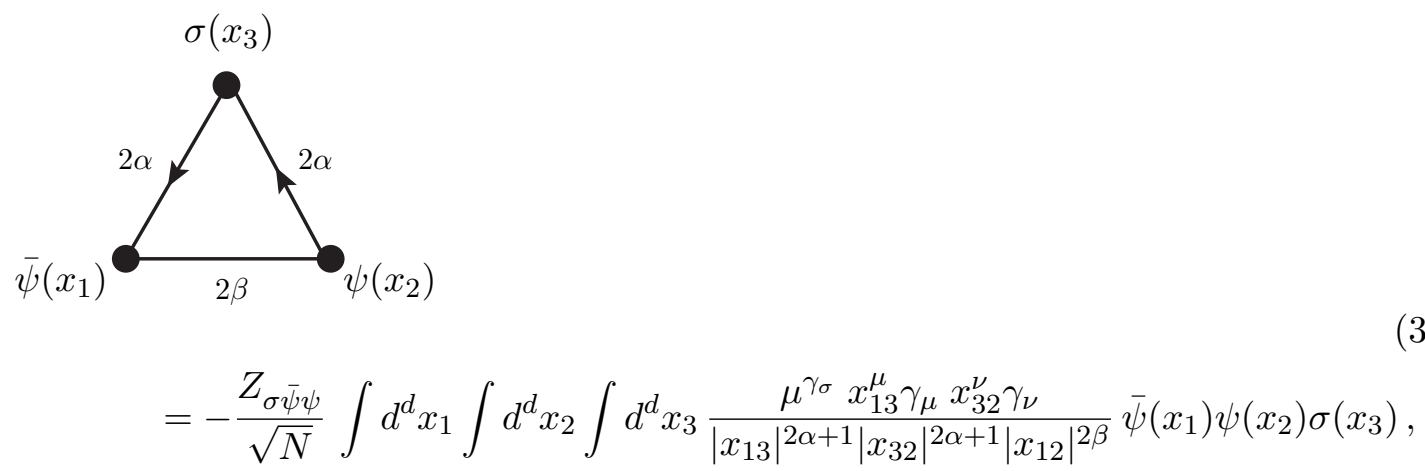

Exponents $2 \alpha, 2 \beta$ of internal lines of the $\sigma \bar{\psi} \psi$ conformal triangle have been chosen so that when the full $\sigma$ and $\psi$ propagators are attached to it, each of the three internal vertices of the triangle are rendered unique:

$$
\alpha=\frac{d-s+1-\gamma_{\sigma}}{2}, \quad \beta=s-1+\frac{\gamma_{\sigma}}{2} .
$$

Performing the resulting three unique integrals then generates the factor of

$$
\begin{aligned}
\text { integrals of }(3.34)= & -\pi^{\frac{3 d}{2}} A\left(s-1+\gamma_{\sigma}\right) V\left(\frac{d-s+1-\gamma_{\sigma}}{2}, \frac{d-s+1-\gamma_{\sigma}}{2}\right) \\
& \times A\left(\frac{d-\gamma_{\sigma}}{2}\right) V\left(\frac{d-s+1}{2}, \frac{s-1+\gamma_{\sigma}}{2}\right) \\
& \times A\left(s-1+\frac{\gamma_{\sigma}}{2}\right) V\left(\frac{d-s+1}{2}, \frac{d-s+1-\gamma_{\sigma}}{2}\right) .
\end{aligned}
$$

Expanding in $1 / N$, we can re-write this factor as

$$
\text { integrals of }(3.34)=u_{\sigma \bar{\psi} \psi}^{(0)}\left(1+\delta u_{\sigma \bar{\psi} \psi}+\mathcal{O}\left(\frac{1}{N^{2}}\right)\right)
$$


where we denoted

$$
\begin{aligned}
u_{\sigma \bar{\psi} \psi}^{(0)} & =-N \frac{\pi^{\frac{3 d}{2}} \Gamma\left(\frac{s}{2}\right)^{2} \Gamma\left(\frac{d}{2}-s+1\right)^{2} \Gamma\left(-\frac{d}{2}+s-1\right)}{\Gamma(s-1)^{2} \Gamma(d-s+1) \Gamma\left(\frac{d-s}{2}+1\right)^{2}}, \\
\delta u_{\sigma \bar{\psi} \psi} & =\frac{1}{N} \frac{\Gamma\left(\frac{s}{2}\right)^{2} \Gamma(d-s+1)\left(2 H_{\frac{d-s}{2}}-3 H_{\frac{d}{2}-s}+\psi^{(0)}\left(\frac{d}{2}\right)+2 \psi^{(0)}\left(\frac{s}{2}\right)-3 \psi^{(0)}(s-1)\right)}{\Gamma\left(\frac{d}{2}\right) \Gamma\left(\frac{d-s}{2}+1\right)^{2} \Gamma\left(-\frac{d}{2}+s-1\right)} .
\end{aligned}
$$

Additionally taking into account the propagator amplitude corrections ${ }^{15}$ one can obtain the three-point function $\langle\sigma \bar{\psi} \psi\rangle$. This calculation will be completed in section 3.4.

Conformal triangle can be calculated at the desired order in $1 / N$ expansion by summing up all contributing diagrams up to the given order. These diagrams include vertex corrections, propagator corrections, and counterterms. Many of these diagrams contain dressed or regularized propagators, rendering them non-integrable via the method of uniqueness. In [31] the background field method was proposed, that allows to easily calculate conformal triangles by using the propagator merging relations. ${ }^{16}$ In such an approach, one of the fields is formally fixed to a non-dynamical background value. For instance, one can fix the Hubbard-Stratonovich field to a constant $\sigma \equiv \bar{\sigma}$, while attaching the full fermionic propagators to the $\sigma \bar{\psi} \psi$ conformal triangle. The conformal triangle can then be solved for, by considering two equivalent forms of writing down the fermionic propagator $\left.\langle\psi \bar{\psi}\rangle\right|_{\bar{\sigma}}$ in the Hubbard-Stratonovich background $\bar{\sigma}$ :
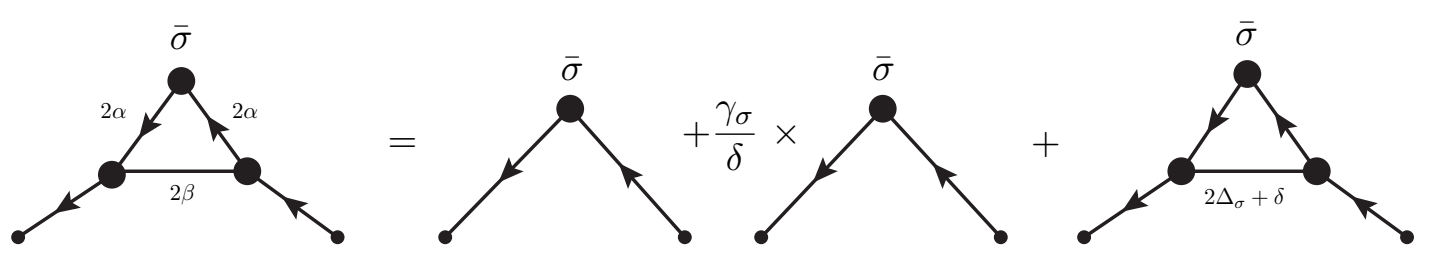

On the 1.h.s. of $(3.40)$ we obtain ${ }^{17}$

$$
\frac{\text { l.h.s. of }(3.40)}{\bar{\sigma} C_{\psi}^{2}\left(1+A_{\psi}\right)^{2}}=\frac{Z_{\sigma \bar{\psi} \psi}^{(0)}}{\sqrt{N}} u_{\sigma \bar{\psi} \psi}^{(0)}\left(1+\delta Z_{\sigma \bar{\psi} \psi}+\delta u_{\sigma \bar{\psi} \psi}+\gamma_{\sigma} \log (\mu|x|)\right)
$$

where we have also expanded amplitude of the conformal triangle in $1 / N$,

$$
Z_{\sigma \bar{\psi} \psi}=Z_{\sigma \bar{\psi} \psi}^{(0)}\left(1+\delta Z_{\sigma \bar{\psi} \psi}\right)
$$

For the first term on the r.h.s. of (3.40) we derive

$$
\frac{\left.\langle\psi \bar{\psi}\rangle\right|_{\bar{\sigma}}}{\bar{\sigma} C_{\psi}^{2}\left(1+A_{\psi}\right)^{2}} \supset-\frac{C_{\sigma \bar{\psi} \psi}^{(0)}}{C_{\sigma}^{\frac{1}{2}} C_{\psi}} \frac{1}{|x|^{d-2 s+2}}
$$

\footnotetext{
${ }^{15}$ Importance of the propagator amplitude corrections in calculation of three-point functions in large- $N$ vector models was first emphasized in [39].

${ }^{16}$ The background field method was recently used to calculate CFT data in the long-range $O(N)$ vector model [16].

${ }^{17}$ It is convenient to factor out $\bar{\sigma} C_{\psi}^{2}\left(1+A_{\psi}\right)^{2}$ from each term on both sides of (3.40). Integrals over vertices of the diagram on the l.h.s. of (3.40) produces the factor equal to the negative of (3.37).
} 
where for the future convenience we introduced

$$
C_{\sigma \bar{\psi} \psi}^{(0)}=-\frac{1}{\sqrt{N}} C_{\sigma}^{\frac{1}{2}} C_{\psi} \pi^{\frac{d}{2}} A(s-1) V\left(\frac{d-s+1}{2}, \frac{d-s+1}{2}\right) .
$$

Comparing (3.43) with the leading-order contribution to (3.41), we obtain

$$
Z_{\sigma \bar{\psi} \psi}^{(0)}=-\sqrt{N} \frac{C_{\sigma \bar{\psi} \psi}^{(0)}}{C_{\sigma}^{\frac{1}{2}} C_{\psi} u_{\sigma \bar{\psi} \psi}^{(0)}}=-\frac{1}{N} \frac{\Gamma(s-1) \Gamma(d-s+1)}{\pi^{d} \Gamma\left(\frac{d}{2}-s+1\right) \Gamma\left(-\frac{d}{2}+s-1\right)} .
$$

At the same time, the sub-leading order contributions to (3.41) are to be compared to the regularized vertex correction diagram, represented by the last term in the r.h.s. of (3.40), given by

$$
\begin{aligned}
\frac{\left.\langle\psi \bar{\psi}\rangle\right|_{\bar{\sigma}}}{\overline{\bar{\sigma}} C_{\psi}^{2}\left(1+A_{\psi}\right)^{2}} \supset & -\frac{1}{N^{\frac{3}{2}}} \pi^{\frac{3 d}{2}} C_{\psi}^{2} C_{\sigma} A(s-1) V\left(\frac{d-s+1}{2}, \frac{d-s+1}{2}\right) A\left(\frac{d+\delta}{2}\right) \\
& \times V\left(\frac{d-s+1}{2}, \frac{s-1-\delta}{2}\right) A\left(s-1-\frac{\delta}{2}\right) \\
& \times V\left(\frac{d-s+1}{2}, \frac{d-s+1+\delta}{2}\right) \frac{\mu^{-\delta}}{|x|^{d-2 s+2+\delta}} .
\end{aligned}
$$

Expanding around $\delta=0$, we notice that the pure pole divergence $1 / \delta$ is subtracted by the counterterm diagram in the r.h.s. of (3.40), while the finite part assumes the form

$$
\frac{\left.\langle\psi \bar{\psi}\rangle\right|_{\bar{\sigma}}}{\bar{\sigma} C_{\psi}^{2}\left(1+A_{\psi}\right)^{2}} \supset-\frac{C_{\sigma \bar{\psi} \psi}^{(0)}}{C_{\sigma}^{\frac{1}{2}} C_{\psi}}\left(r_{\sigma \bar{\psi} \psi}+\gamma_{\sigma} \log (\mu|x|)\right),
$$

where we denoted

$$
r_{\sigma \bar{\psi} \psi}=\frac{1}{N} \frac{\Gamma\left(\frac{s}{2}\right)^{2} \Gamma(d-s+1)\left(\psi^{(0)}\left(\frac{d}{2}\right)-H_{\frac{d}{2}-s}-H_{s-2}+\gamma\right)}{\Gamma\left(\frac{d}{2}\right) \Gamma\left(\frac{d-s}{2}+1\right)^{2} \Gamma\left(-\frac{d}{2}+s-1\right)},
$$

where $\gamma$ is the Euler constant. Comparing (3.47) with the sub-leading contribution to (3.41), while using (3.45), we notice that the anomalous dimension logarithm terms match, while the relative correction to amplitude of the conformal triangle can be expressed as

$$
\begin{aligned}
\delta Z_{\sigma \bar{\psi} \psi} & =r_{\sigma \bar{\psi} \psi}-\delta u_{\sigma \bar{\psi} \psi} \\
& =\frac{1}{N} \frac{2 \Gamma\left(\frac{s}{2}\right)^{2} \Gamma(d-s+1)\left(H_{\frac{d}{2}-s}-H_{\frac{d-s}{2}}-\psi^{(0)}\left(\frac{s}{2}\right)+\psi^{(0)}(s-1)\right)}{\Gamma\left(\frac{d}{2}\right) \Gamma\left(\frac{d-s}{2}+1\right)^{2} \Gamma\left(-\frac{d}{2}+s-1\right)} .
\end{aligned}
$$

\section{$3.4\langle\sigma \bar{\psi} \psi\rangle$}

At the leading order in $1 / N$ expansion, the three-point function $\langle\sigma \bar{\psi} \psi\rangle$ is determined by the tree-level diagram

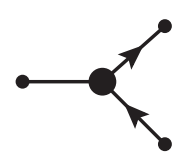


This diagram is easy to evaluate taking one conformal integral using the uniqueness relation. This gives

$$
\left.\left\langle\sigma\left(x_{1}\right) \bar{\psi}\left(x_{2}\right) \psi\left(x_{3}\right)\right\rangle\right|_{\text {normalized }}=\frac{C_{\sigma \bar{\psi} \psi}^{(0)} x_{12}^{\mu} \gamma_{\mu} x_{31}^{\nu} \gamma_{\nu}}{\left(\left|x_{12}\right|\left|x_{13}\right|\right)^{s}\left|x_{23}\right|^{d-2 s+2}},
$$

where the amplitude $C_{\sigma \bar{\psi} \psi}^{(0)}$ is given by (3.44). Following the same conventions throughout this paper, all three-point functions are written down for operators whose propagators in position space have been normalized to unity.

To incorporate $1 / N$ contributions due to the vertex and propagator corrections, we can use $\sigma \bar{\psi} \psi$ conformal triangle, calculated in section 3.4, and relative propagator amplitude corrections, calculated in section 3.1 and section 3.2. This gives the full three-point function

$$
\left.\left\langle\sigma\left(x_{1}\right) \bar{\psi}\left(x_{2}\right) \psi\left(x_{3}\right)\right\rangle\right|_{\text {normalized }}=\frac{C_{\sigma \bar{\psi} \psi}^{(0)}\left(1+\delta C_{\sigma \bar{\psi} \psi}\right) x_{12}^{\mu} \gamma_{\mu} x_{31}^{\nu} \gamma_{\nu}}{\left(\left|x_{12}\right|\left|x_{13}\right|\right)^{s+\gamma_{\sigma}}\left|x_{23}\right|^{d-2 s+2-\gamma_{\sigma}}},
$$

Attaching full propagator legs to the conformal triangle and taking integrals over the unique vertices, we obtain:

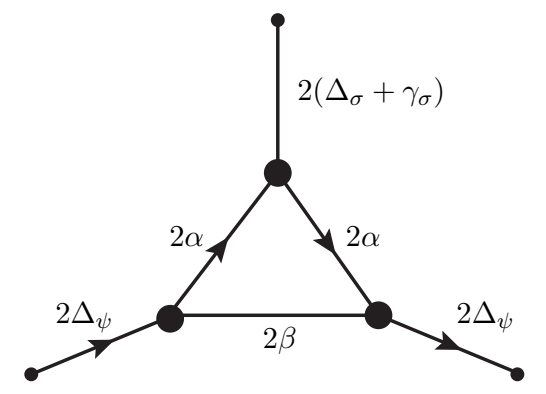

Therefore the relative correction to the three-point function amplitude is given by

$$
\begin{aligned}
\delta C_{\sigma \bar{\psi} \psi}= & \delta Z_{\sigma \bar{\psi} \psi}+\delta u_{\sigma \bar{\psi} \psi}+\frac{A_{\sigma}}{2}+A_{\psi} \\
= & -\frac{1}{N} \frac{\Gamma\left(\frac{s}{2}\right)^{2} \Gamma(d-s+1)}{\Gamma\left(\frac{d}{2}\right) \Gamma\left(\frac{d-s}{2}+1\right)^{2} \Gamma\left(-\frac{d}{2}+s-1\right)}\left(H_{\frac{d}{2}-s}-H_{\frac{d-s}{2}}+H_{d-s}\right. \\
& \left.+\psi^{(0)}\left(-\frac{d}{2}+s-1\right)-\psi^{(0)}\left(\frac{d}{2}\right)-\psi^{(0)}\left(\frac{s}{2}\right)+\psi^{(0)}(s-1)\right)+\mathcal{O}\left(\frac{1}{N^{2}}\right)
\end{aligned}
$$

We close this section by expanding the leading and next-to-leading contributions to the $\langle\sigma \bar{\psi} \psi\rangle$ OPE coefficients at $d=4-\epsilon_{2}, s=2-\epsilon_{2} / 2+\epsilon_{1}$ to leading order in $\epsilon_{1,2}$,

$$
\begin{aligned}
\left.C_{\sigma \bar{\psi} \psi}^{(0)}\right|_{d=4} & =-\sqrt{\frac{2 \epsilon_{1}}{N}}+\mathcal{O}\left(\epsilon_{1,2}^{\frac{3}{2}}, \frac{1}{N^{\frac{3}{2}}}\right), \\
\left.\delta C_{\sigma \bar{\psi} \psi}\right|_{d=4} & =-\frac{2}{N}+\mathcal{O}\left(\epsilon_{1,2}, \frac{1}{N^{2}}\right) .
\end{aligned}
$$

These expressions will be compared with their counterparts in the Gross-Neveu-Yukawa model in section 4 , where we will perform perturbative $\epsilon$-expansion near $d=4, s=2$, to the order $\mathcal{O}\left(\sqrt{\epsilon_{1,2}}\right)$. 


\subsection{Continuity of CFT data at $s=s_{\star}$}

In this section we have derived various CFT data, including scaling dimensions of the fields $\psi, \sigma$, and the OPE coefficient $\langle\sigma \bar{\psi} \psi\rangle$ in the critical long-range Gross-Neveu model (3.13) at its interacting UV fixed point, working at the next-to-leading order in $1 / N$ expansion. We are now going to check continuity of this CFT data across the long-range-short-range crossover point $s_{\star}$. This will serve as a non-trivial consistency check for our results.

Recall that $s_{\star}$ is defined as the threshold value of the long-range exponent parameter $s$, above which the bi-local fermionic kinetic term (3.1) is irrelevant compared to the local (short-range) Dirac kinetic term. By calculating scaling dimension of (3.1) in the shortrange model, we obtain $s_{\star}=2-2 \gamma_{\hat{\psi}}$, where anomalous dimension of fermionic field in the short-range critical Gross-Neveu model is given by (2.1). This immediately guarantees that the fermionic scaling dimension is continuous,

$$
\left.[\psi]_{\mathrm{UV}}\right|_{s=s_{\star}}=\frac{d-s_{\star}+1}{2}=\frac{d-1}{2}+\gamma_{\hat{\psi}}=[\hat{\psi}]_{\mathrm{UV}} .
$$

Continuity of the full scaling dimension of the Hubbard-Stratonovich field can be established by noticing that the difference between divergent graphs contributing to the $\langle\sigma \sigma\rangle$ propagator in the long-range model and the $\langle\hat{\sigma} \hat{\sigma}\rangle$ propagator in the short-range model can be traced back to the vanishing anomalous dimension of the fermion in the former, and therefore absence of the corresponding diagram, contributing $2 \gamma_{\hat{\psi}}$. This leads to the relation

$$
\left.\gamma_{\sigma}\right|_{s=2}=\gamma_{\hat{\sigma}}+2 \gamma_{\hat{\psi}}
$$

that can be verified explicitly using $(2.1),(2.4),(3.29)$. Here we have used $s_{\star}=2+\mathcal{O}(1 / N)$, and expanded our expressions to the next-to-leading order in $1 / N$, that defined the regime of validity of our results. We can then derive

$$
\left.[\sigma]_{\mathrm{UV}}\right|_{s=s_{\star}}=s_{\star}-1+\left.\gamma_{\sigma}\right|_{s=2}=1+\left(\left.\gamma_{\sigma}\right|_{s=2}-2 \gamma_{\hat{\psi}}\right)=[\hat{\sigma}]_{\mathrm{UV}}
$$

Finally, continuity of the $\langle\sigma \bar{\psi} \psi\rangle$ OPE coefficient across the long-range-short-range crossover $s_{\star}$ can be established by explicitly verifying validity of the following identities: ${ }^{18}$

$$
\begin{gathered}
\left.C_{\sigma \bar{\psi} \psi}^{(0)}\right|_{s=2}=C_{\hat{\sigma} \hat{\psi} \hat{\psi}}^{(0)}, \\
\left.\left(C_{\sigma \bar{\psi} \psi}^{(0)} \delta C_{\sigma \bar{\psi} \psi}-2 \gamma_{\hat{\psi}} \frac{\partial C_{\sigma \bar{\psi} \psi}^{(0)}}{\partial s}\right)\right|_{s=2}=C_{\hat{\sigma} \hat{\psi} \hat{\psi}}^{(0)} \delta C_{\hat{\sigma} \overline{\hat{\psi}} \hat{\psi}}
\end{gathered}
$$

\section{Long-range Gross-Neveu-Yukawa model}

In this section we will construct the long-range version of the Gross-Neveu-Yukawa model [2], describing dynamics of the Dirac fermions $\theta^{i}, i=1, \ldots, n$, in the fundamental representation of the $\mathrm{U}(n)$ symmetry group, coupled to the pseudo-scalar $\phi$. Our goal is

\footnotetext{
${ }^{18}$ Analogous consistency checks of continuity of CFT data across long-range-short-range crossover in $O(N)$ vector models have recently been performed in [16].
} 
to develop the model that flows to an IR-stable interacting fixed point that is equivalent to the critical regime of the long-range Gross-Neveu model (3.13).

Establishing correspondence between the models begins with the match of d.o.f.,

$$
\psi^{i} \leftrightarrow \theta^{i}, \quad i=1, \ldots, n, \quad \sigma \leftrightarrow \phi .
$$

The most non-trivial part of the proposed critical duality will manifest itself in the equivalency of the CFT data of these two models. To this end, we start by noticing that since the scaling dimension of the fermionic field $\psi^{i}$ in the long-range Gross-Neveu model (3.13) is fixed exactly to (3.2), we want to ensure that the scaling dimension of the fermionic field $\theta^{i}$ in the long-range Gross-Neveu-Yukawa model is similarly non-renormalized. This can be achieved by choosing the bi-local fermionic kinetic term

$$
S_{\mathrm{LR} \operatorname{Dirac}}[\theta]=C_{f}(s) \int d^{d} x \int d^{d} y \bar{\theta}^{i}(x) \gamma^{\mu} \theta^{i}(y) \frac{(x-y)^{\mu}}{|x-y|^{d+s}},
$$

for the fermion $\theta^{i}$. The pre-factor $C_{f}(s)$ is given by (3.4), ensuring that the momentum space propagator is canonically normalized. In position space this propagator is given by

$$
\langle\theta(x) \bar{\theta}(0)\rangle=C_{\theta} \frac{\gamma^{\mu} x_{\mu}}{|x|^{2 \Delta_{\theta}+1}},
$$

where the scaling dimension is given by

$$
\Delta_{\theta}=\frac{d-s+1}{2},
$$

while the free propagator amplitude is defined as

$$
C_{\theta}=\frac{\Gamma\left(\frac{d-s}{2}+1\right)}{2^{s-1} \pi^{\frac{d}{2}} \Gamma\left(\frac{s}{2}\right)} .
$$

We then couple the model (4.2) to the pseudo-scalar field $\phi$,

$$
S_{\mathrm{LR} \mathrm{GNY}}=S_{\mathrm{LR} \operatorname{Dirac}}[\theta]+\int d^{d} x\left(\frac{1}{2}(\partial \phi)^{2}+g \phi \bar{\theta} \theta+\frac{h}{24} \phi^{4}\right) .
$$

Notice that analogously to the symmetry (3.14) of the long-range Gross-Neveu model, the action (4.6) enjoys the $\mathbb{Z}_{2}$ symmetry

$$
\begin{aligned}
\left(x^{1}, \ldots, x^{a-1}, x^{a}, x^{a+1}, \ldots, x^{d}\right) & \rightarrow\left(x^{1}, \ldots, x^{a-1},-x^{a}, x^{a+1}, \ldots, x^{d}\right), \\
\phi \rightarrow-\phi, \quad \theta & \rightarrow \gamma^{a} \theta, \quad \bar{\theta} \rightarrow-\bar{\theta} \gamma^{a}
\end{aligned}
$$

defined for any given $a=1, \ldots, d$. Additionally, since we can redefine $\phi \rightarrow-\phi$, this implies that every non-trivial fixed point value $g_{\star}$ is paired up with a physically equivalent fixed point $-g_{\star}$.

In the free regime, the propagator of the scalar field $\phi$ is given by

$$
\langle\phi(x) \phi(0)\rangle=\frac{C_{\phi}}{|x|^{2 \Delta_{\phi}}},
$$


where the scaling dimension is given by

$$
\Delta_{\phi}=\frac{d}{2}-1
$$

while the free propagator amplitude is

$$
C_{\phi}=\frac{\Gamma\left(\frac{d}{2}-1\right)}{4 \pi^{\frac{d}{2}}} .
$$

Near the free regime, $g=0, h=0$, the coupling constants therefore have dimensions

$$
[g]_{\text {free }}=s-\frac{d}{2}, \quad[h]_{\text {free }}=4-d .
$$

Choosing

$$
d=4-\epsilon_{2}, \quad s=\frac{d}{2}+\epsilon_{1}=2-\frac{\epsilon_{2}}{2}+\epsilon_{1},
$$

we can make the couplings $g, h$ slightly relevant near the UV. We then have

$$
\Delta_{\theta}=\frac{3}{2}-\frac{\epsilon_{1}}{2}-\frac{\epsilon_{2}}{4}, \quad \Delta_{\phi}=1-\frac{\epsilon_{2}}{2} .
$$

Let us now redefine the coupling constants to be dimensionless,

$$
S_{\mathrm{LR} \mathrm{GNY}}=S_{\mathrm{LR} \operatorname{Dirac}}[\theta]+\int d^{d} x\left(\frac{1}{2}(\partial \phi)^{2}+g \mu^{\epsilon_{1}} \phi \bar{\theta} \theta+\frac{h \mu^{\epsilon_{2}}}{24} \phi^{4}\right),
$$

where $\mu$ is an arbitrary RG scale. The relevant interaction in the UV activates an RG flow, that brings the theory to an interacting Wilson-Fisher fixed point $\left(g_{\star}, h_{\star}\right)$ in the IR, that can be found perturbatively using $\epsilon$-expansion. Our goal in this section is to find this fixed point and study the corresponding critical theory.

While we will carry out our calculation at the next-to-leading order in $\epsilon$-expansion, our results in this section are going to be exact in $1 / N$. However, since we are largely motivated by the desire to compare the corresponding critical theory at the fixed point with the critical long-range Gross-Neveu model, that we discussed in section 3, we will subsequently perform algebraic expansion of our results in $1 / N$. Specifically, the fixedpoint values of the dimensionless coupling constants can be represented as

$$
\begin{aligned}
& g_{\star}=g_{0}\left(1+\frac{g_{1}}{N}+\mathcal{O}\left(\frac{1}{N^{2}}\right)\right), \\
& h_{\star}=h_{0}\left(1+\frac{h_{1}}{N}+\mathcal{O}\left(\frac{1}{N^{2}}\right)\right) .
\end{aligned}
$$

From the action (4.14) it is clear that $\theta^{i} \sim \mathcal{O}\left(1 / N^{0}\right), \phi \sim \mathcal{O}(\sqrt{N})$, and therefore

$$
g_{0} \sim \mathcal{O}\left(\frac{1}{\sqrt{N}}\right), \quad h_{0} \sim \mathcal{O}\left(\frac{1}{N}\right)
$$

Using the Callan-Symanzik equation

$$
\left(\mu \frac{\partial}{\partial \mu}+\beta_{g} \frac{\partial}{\partial g}+\gamma_{\phi}\right)\langle\phi \bar{\theta} \theta\rangle=0
$$


where we took into account $\gamma_{\theta}=0$, and expanded to the leading order in $1 / N$, we then obtain

$$
\gamma_{\phi}=\epsilon_{1}+\mathcal{O}\left(\frac{1}{N}\right)
$$

and as a result using $(4.12),(4.13)$ we obtain $[\phi]_{\mathrm{IR}}=s-1+\mathcal{O}\left(\frac{1}{N}\right)$. While below in this section we will calculate $\gamma_{\phi}$ at leading order in $\epsilon$-expansion but to all orders in $1 / N$, we can already notice that such a large- $N$ behavior of $[\phi]_{\text {IR }}$ matches the scaling dimension (3.20) of the Hubbard-Stratonovich field of the critical long-range Gross-Neveu model. Moreover, it indicates that the quartic and the local kinetic term for the field $\phi$ in the action (4.14), that naively distinguish it from the long-range Gross-Neveu model (3.13) in the UV, are in fact irrelevant in the IR fixed point of the model (4.14).

We will carry out perturbative $\epsilon$-expansion in position space. The Feynman rules that we are going to be using in this section for the free fermionic and scalar propagator, and the cubic interaction vertex, are given by
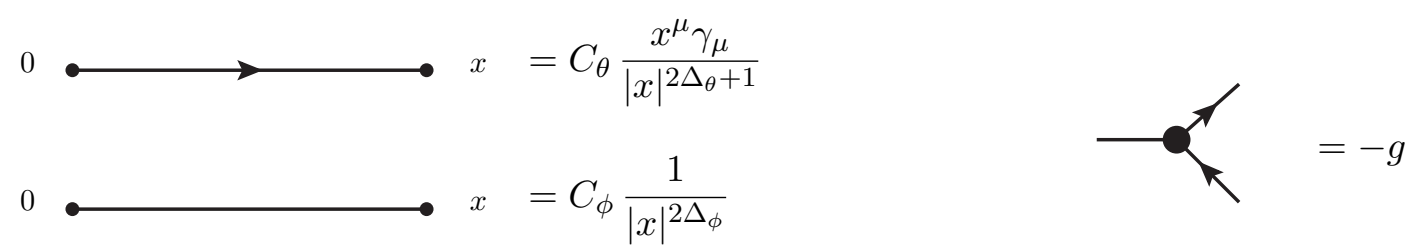

For fermionic and scalar lines with explicitly specified exponents we will define the corresponding amplitudes to be equal to unity. Together with the quartic interaction vertex, the corresponding Feynman rules are given by
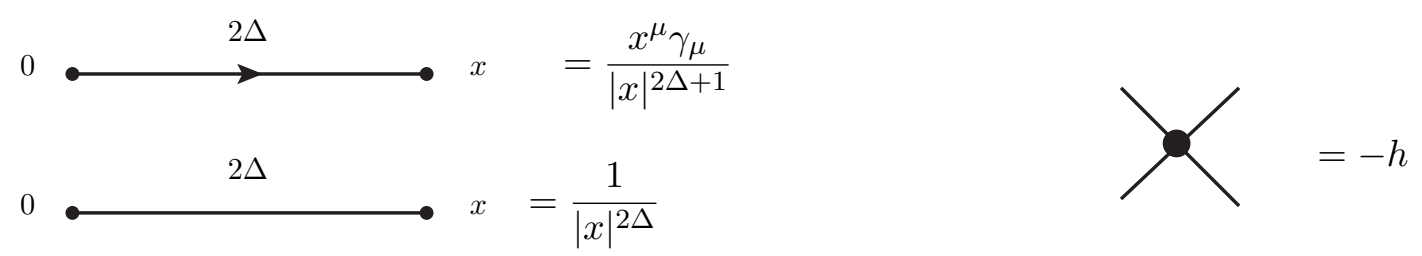

\section{$4.1\langle\phi \phi\rangle$}

At the leading order in $\epsilon$-expansion, the $\langle\phi \phi\rangle$ propagator is given by the free field expression (4.8). One-loop correction to this expression is determined by the following diagram: ${ }^{19}$

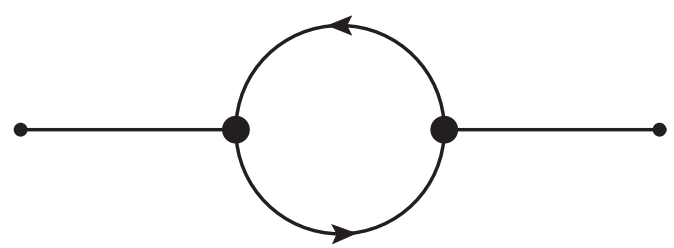

\footnotetext{
${ }^{19}$ We argue that the two-loop $\mathcal{O}\left(h^{2}\right)$ diagram is of a sub-leading order $\mathcal{O}\left(\epsilon_{1}^{2}\right)$. This is analogous to the short-range Gross-Neveu-Yukawa model, where at the Wilson-Fisher fixed point in $d=4-\epsilon$ dimensions the leading order couplings behave as $g=\mathcal{O}(\sqrt{\epsilon}), h=\mathcal{O}(\epsilon)$.
} 
In this diagram, the Feynman rule for the fermionic loop contributes the factor of -1 . Combining two fermionic propagators forming a loop renders another factor of -1 , as we reviewed in appendix A. Using the propagator merging relations, and combining the resulting expression with the leading order propagator (4.8), we obtain

$$
\begin{aligned}
\langle\phi(x) \phi(0)\rangle= & \frac{C_{\phi}}{|x|^{2-\epsilon_{2}}}\left(1+g_{\star}^{2} N C_{\theta}^{2} C_{\phi} U\left(1-\frac{\epsilon_{2}}{2}, 3-\epsilon_{1}-\frac{\epsilon_{2}}{2}, \epsilon_{1}\right)\right. \\
& \left.\times U\left(1-\frac{\epsilon_{2}}{2}, 2-\epsilon_{1}-\frac{\epsilon_{2}}{2}, 1+\epsilon_{1}\right)(\mu|x|)^{2 \epsilon_{1}}\right) \\
& \rightarrow \frac{C_{\phi}}{|x|^{2-\epsilon_{2}}}\left(1-\frac{N g_{\star}^{2}}{16 \pi^{2}} \log (\mu|x|)\right),
\end{aligned}
$$

where in the last line we performed expansion in $\epsilon_{1}$, collected leading order terms in front of the logarithm, and discarded the $1 / \epsilon_{1}$ term. ${ }^{20}$ Consequently, anomalous dimension of the field $\phi$ at the IR f.p. is given by

$$
\gamma_{\phi}=\frac{N g_{\star}^{2}}{32 \pi^{2}}+\mathcal{O}\left(\epsilon_{1}^{2}\right)
$$

\section{$4.2\langle\phi \bar{\theta} \theta\rangle$}

We now proceed to calculation of the three-point function $\langle\phi \bar{\theta} \theta\rangle$ up to the one-loop order. At the leading order it is given by the diagram

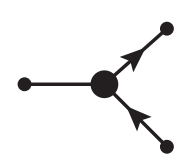

The corresponding contribution to the three-point function reads ${ }^{21}$

$$
\left.\left\langle\phi\left(x_{1}\right) \bar{\theta}\left(x_{2}\right) \theta\left(x_{3}\right)\right\rangle\right|_{\text {normalized }}=\frac{C_{\phi \bar{\theta} \theta} x_{12}^{\mu} \gamma_{\mu} x_{31}^{\nu} \gamma_{\mu}}{\left(\left|x_{12}\right|\left|x_{13}\right|\left|x_{23}\right|\right)^{2}},
$$

where

$$
C_{\phi \bar{\theta} \theta}=-\left.g_{\star} C_{\phi}^{\frac{1}{2}} C_{\theta} \pi^{\frac{d}{2}} A(1) V\left(\frac{d-1}{2}, \frac{d-1}{2}\right)\right|_{d=4, s=2}+\mathcal{O}\left(g_{\star}^{3}\right) .
$$

The motivation behind such a choice is that it provides us with universal conventions allowing us to compare the corresponding OPE coefficients in different models.

${ }^{20}$ These pure divergencies can be absorbed into the wave-function

$$
\phi \rightarrow \sqrt{1+\frac{\gamma_{\phi}}{\epsilon_{1}}} \phi
$$

We keep renormalizations implicit here and everywhere else in this section.

${ }^{21}$ At the leading order in $\epsilon$-expansion one drops $\mathcal{O}(\epsilon)$ contributions to the exponents of $\left|x_{i j}\right|$. 
At the next-to-leading order in $\epsilon$-expansion we have a contribution from the following vertex correction one-loop diagram:

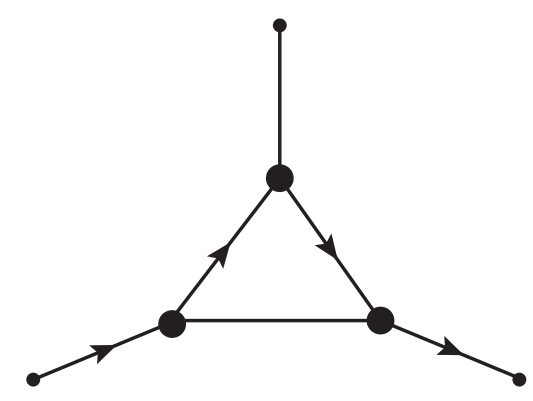

The corresponding term in the three-point function is given by

$$
\left.\left\langle\phi\left(x_{1}\right) \bar{\theta}\left(x_{2}\right) \theta\left(x_{3}\right)\right\rangle\right|_{\text {normalized }}=\left(-g_{\star}\right)^{3} C_{\phi}^{\frac{3}{2}} C_{\theta}^{3} V\left(\mu_{0}\right),
$$

where $V\left(\mu_{0}\right)$ is determined by the regularized diagram

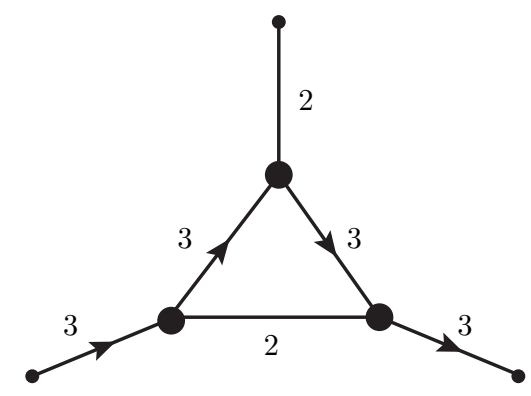

This diagram is logarithmically divergent. It can be regularized by imposing the UV cut-off $|x| \geq 1 / \mu_{0}$, and subtracting the divergent term $\log \left(\mu_{0}\right)$ with the vertex counterterm, at the cost of introducing an RG scale $\mu$ :

$$
\left.\int_{1 / \mu_{0}} \frac{d|x|}{|x|}\right|_{\text {regularized }}=\log (\mu)
$$

The integrals over the other two vertices are finite, and can be taken using the star-triangle relation, reviewed in appendix $\mathrm{A}$. We then arrive at

$$
V(\mu)=-\left.2 \pi^{4} A(1) V\left(\frac{3}{2}, \frac{3}{2}\right) U\left(1, \frac{3}{2}, \frac{3}{2}\right)\right|_{d=4, s=2} \frac{x_{12}^{\mu} \gamma_{\mu} x_{31}^{\nu} \gamma_{\mu}}{\left(\left|x_{12}\right|\left|x_{13} \| x_{23}\right|\right)^{2}} \log (\mu)
$$

Finally, dressing the $\phi$ leg of the tree-level $\langle\phi \bar{\theta} \theta\rangle$ diagram, we obtain

$$
\left.\left\langle\phi\left(x_{1}\right) \bar{\theta}\left(x_{2}\right) \theta\left(x_{3}\right)\right\rangle\right|_{\text {normalized }}=-2 \gamma_{\phi} \log (\mu) \frac{C_{\phi \bar{\theta} \theta} x_{12}^{\mu} \gamma_{\mu} x_{31}^{\nu} \gamma_{\mu}}{\left(\left|x_{12}\right|\left|x_{13}\right|\left|x_{23}\right|\right)^{2}},
$$


Using the Callan-Symanzik equation

$$
\left(\mu \frac{\partial}{\partial \mu}+\beta_{g} \frac{\partial}{\partial g}+\gamma_{\phi}\right)\left\langle\phi\left(x_{1}\right) \bar{\theta}\left(x_{2}\right) \theta\left(x_{3}\right)\right\rangle=0
$$

we then obtain

$$
\beta_{g}=-\epsilon_{1} g+\frac{N+4}{32 \pi^{2}} g^{3} .
$$

A non-trivial IR fixed point is then achieved $\mathrm{at}^{22}$

$$
g_{\star}=4 \pi \sqrt{\frac{2 \epsilon_{1}}{N+4}} .
$$

Expanding (4.29) in $1 / N$ and plugging it into (4.22), we then obtain the normalized threepoint function amplitude

$$
C_{\phi \bar{\theta} \theta}=-\sqrt{\frac{2 \epsilon_{1}}{N}}\left(1-\frac{2}{N}+\mathcal{O}\left(\frac{1}{N^{2}}\right)\right)+\mathcal{O}\left(\epsilon_{1}^{3 / 2}\right) .
$$

On the other hand, substituting (4.29) into (4.20) and expanding in $1 / N$, we obtain

$$
[\phi]_{\mathrm{IR}}=\Delta_{\phi}+\gamma_{\phi}=1-\frac{\epsilon_{2}}{2}+\epsilon_{1}-\frac{4 \epsilon_{1}}{N}+\mathcal{O}\left(\epsilon_{1}^{2}, \frac{1}{N^{2}}\right)=s-1-\frac{4 \epsilon_{1}}{N}+\mathcal{O}\left(\epsilon_{1}^{2}, \frac{1}{N^{2}}\right),
$$

where we used (4.12), (4.13).

Notice that while our calculation is perturbative in $\epsilon_{1,2}$, it is exact in $1 / N$. However, performing algebraic $1 / N$ expansion allows us to compare the resulting expressions $(4.30),(4.31)$ at the next-to-leading order in $1 / N$ with their counterparts $(3.53),(3.33)$ in the large- $N$ long-range Gross-Neveu model, revealing an exact agreement.

These non-trivial matches of CFT data at the next-to-leading order in $1 / N$ expansion provide a strong supportive evidence in favor of the statement that the UV fixed point of the long-range Gross-Neveu model, and the IR fixed point of the long-range Gross-NeveuYukawa model are in fact described by the same CFT. While our calculations have been performed in $\epsilon$-expansion near $d=4, s=2$ for the Gross-Neveu-Yukawa model, and in $1 / N$ expansion for the Gross-Neveu model, we suggest that it holds true for the entire domain (3.22), (3.23).

\section{$4.3\langle\phi \phi \phi \phi\rangle$}

In this section we are going to use the four-point function $\langle\phi \phi \phi \phi\rangle$ at the next-to-leading order in $\epsilon$-expansion to calculate the fixed-point value of the coupling $h_{\star}$. The leading order contribution to the normalized four-point function is given by the tree-level diagram

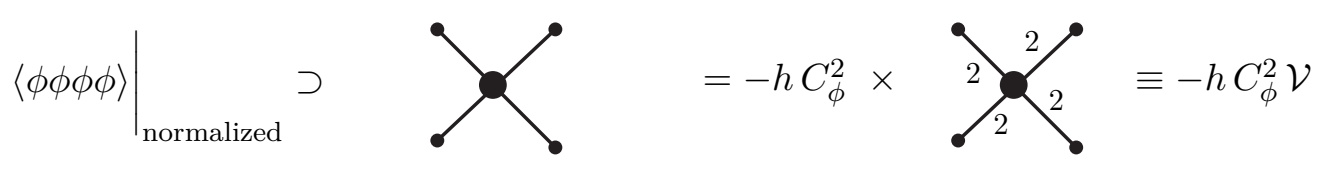

\footnotetext{
${ }^{22}$ Recall that $-g_{\star}$ is a physically equivalent fixed point.
} 
While the four-line vertex on the r.h.s. of this diagrammatic equation is conformal, we do not need to take this integral for our purposes. Instead, we denote it as $\mathcal{V}$ and keep it implicit.

At the next-to-leading order we have contributions due to the dressed $\phi$ legs of the tree-level diagram, as well as two vertex corrections. The former is given by

$$
\left.\langle\phi \phi \phi \phi\rangle\right|_{\text {normalized }} \supset 4 \times\left(-2 \gamma_{\phi}\right)(-h) C_{\phi}^{2} \mathcal{V},
$$

One of the vertex corrections is determined by the diagram

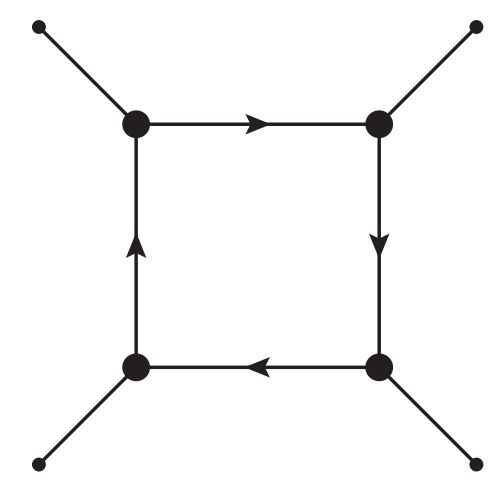

Two of its vertices can be calculated using the uniqueness relation reviewed in appendix A. The third integral is logarithmically divergent, and can be regularized using the UV cutoff, with the divergence being subsequently subtracted using vertex counter-term, resulting in (4.24). This way the diagram is reduced to $\mathcal{V}$, that implicitly contains the fourth integral. Assembling everything together we obtain (here 6 is the symmetry factor and overall minus sign is due to the fermionic loop)

$$
\left.\langle\phi \phi \phi \phi\rangle\right|_{\text {normalized }} \supset-\left.6 N h^{4} C_{\phi}^{2} C_{\theta}^{4}\left(\pi^{\frac{d}{2}} A(1) V\left(\frac{3}{2}, \frac{3}{2}\right)\right)^{2}\right|_{d=4, s=2} 2 \pi^{2} \log (\mu) \mathcal{V} .
$$

The other vertex correction diagram contributing to $\langle\phi \phi \phi \phi\rangle$ at the next-to-leading order in $\epsilon$-expansion is given by

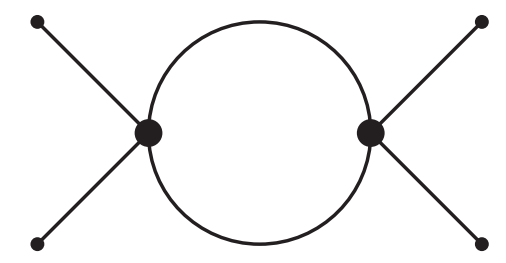

The corresponding contribution has the form (here $3 / 2$ is the symmetry factor)

$$
\left.\langle\phi \phi \phi \phi\rangle\right|_{\text {normalized }} \supset \frac{3}{2} g^{2} C_{\phi}^{4} 2 \pi^{2} \log (\mu) \mathcal{V},
$$


where we regularized one of the integrals. The common factor $\mathcal{V}$ cancels out from the Callan-Symanzik equation,

$$
\left(\mu \frac{\partial}{\partial \mu}+\beta_{h} \frac{\partial}{\partial h}+4 \gamma_{\phi}\right)\langle\phi \phi \phi \phi\rangle=0
$$

resulting in

$$
\beta_{h}=-\epsilon_{2} h+\frac{2 N g^{2}\left(h-6 g^{2}\right)+3 h^{2}}{16 \pi^{2}} .
$$

The corresponding IR stable fixed point is given by

$$
h_{\star}=\frac{8}{3} \pi^{2}\left(\sqrt{\frac{16 N(N+36) \epsilon_{1}^{2}}{(N+4)^{2}}-\frac{8 N \epsilon_{1} \epsilon_{2}}{N+4}+\epsilon_{2}^{2}}-\frac{4 N \epsilon_{1}}{N+4}+\epsilon_{2}\right) .
$$

\section{Discussion}

In this paper we have constructed new long-range Gross-Neveu and Gross-Neveu-Yukawa fermionic models. For the choice of parameters $d / 2<s<\min \left(d / 2+1, s_{\star}\right), 1<d<4$, we demonstrated that these models flow to critical regimes (in the UV and the IR respectively), and matched CFT data of these models within the overlapping regimes of validity. Our paper therefore furnishes a non-trivial generalization between the short-range Gross-Neveu and Gross-Neveu-Yukawa models.

Conformal structure of the three-point function $\langle\phi \bar{\theta} \theta\rangle$ in the long-range Gross-NeveuYukawa model indicates that its fixed point enjoys the full conformal symmetry, at least at the order $\mathcal{O}\left(\sqrt{\epsilon_{1,2}}\right)$ at which we have performed explicit calculations. Additionally, one can check the two-point functions that must vanish if the full conformal symmetry is valid. For instance, the two-point function $\left\langle\phi\left(x_{1}\right) \bar{\psi} \psi\left(x_{2}\right)\right\rangle$ vanishes up to a contact term, and the correlator $\left\langle\phi^{2}\left(x_{1}\right) \phi^{4}\left(x_{2}\right)\right\rangle$ is zero at the considered order in $\epsilon$-expansion. Due to the critical duality argument, provided in this paper, this implies that the critical long-range Gross-Neveu model is also in fact described by a CFT. It would be interesting to see how conformal symmetry at the long-range fixed point of the considered fermionic models can be established analogously to the arguments of [19-22, 29].

As we reviewed in section 1 , the long-range critical vector model can be found at the end of an RG flow, triggered by coupling the critical short-range vector model to a generalized free field of dimension $(d+s) / 2[19,20]$. We suggest that similarly one can couple the short-range Gross-Neveu model to a long-range fermion $\chi$ of dimension $\Delta_{\chi}=(d+s-1) / 2$,

$$
\hat{S}=\left.S_{\mathrm{LR} \operatorname{Dirac}}[\chi]\right|_{2-s}+\lambda \int d^{d} x(\hat{\bar{\psi}} \chi+\bar{\chi} \hat{\psi}) .
$$

Near $s=s_{\star}$ we therefore obtain $[\lambda]=s_{\star}-s$, where $s_{\star}=2-2 \gamma_{\hat{\psi}}$ defines the long-rangeshort-range crossover threshold. For small $\delta=s_{\star}-s$ one can therefore study the model perturbatively in $\lambda$.

Long-range vector models have recently been used in systems exhibiting persistent symmetry breaking [45]. ${ }^{23}$ While these systems have so far been considered to be exclusively bosonic, it would be interesting to see what role the long-range fermions play in models manifesting persistent symmetry breaking.

\footnotetext{
${ }^{23}$ See also $[46,47]$ for recent work on persistent symmetry breaking in vector models.
} 


\section{Acknowledgments}

The work of N.C. and R.S. is partially supported by the Binational Science Foundation (grant No. 2016186), the Israeli Science Foundation Center of Excellence (grant No. 2289/18), and by the Quantum Universe I-CORE program of the Israel Planning and Budgeting Committee (grant No. 1937/12). The work of N.C. is partially supported by Yuri Milner scholarship. The work of S.C. is supported by the Infosys Endowment for the study of the Quantum Structure of Spacetime. R.S. would like to thank CPHT at Ecole Polytechnique, France for hospitality during the course of this work. The work of M.G. is supported by DOE grant DE-SC0011842.

\section{A Some useful identities}

In this appendix we collect some known expressions and identities, that are useful to carry out perturbation theory calculations in position space [30, 48, 49] (see also [50] for a recent review).

Loop diagram in position space are simply additive:

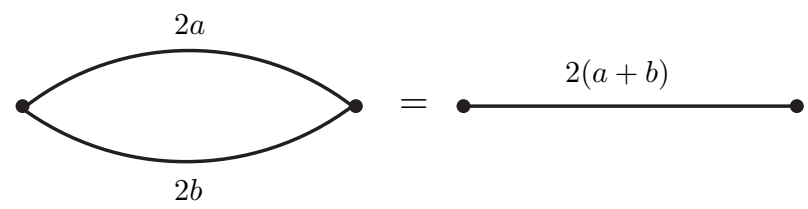

When fermions are involved, we obtain
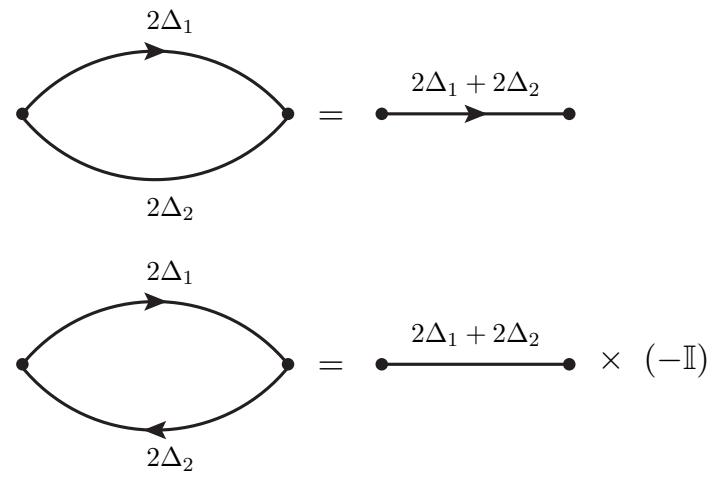

The propagator merging relation identity is given by

$$
\int d^{d} x_{2} \frac{1}{\left|x_{2}\right|^{2 a}\left|x_{1}-x_{2}\right|^{2 b}}=U(a, b, d-a-b) \frac{1}{\left|x_{1}\right|^{2 a+2 b-d}},
$$

where we defined

$$
U(a, b, c)=\pi^{\frac{d}{2}} A(a) A(b) A(c) .
$$

Here we have introduced

$$
A(x)=\frac{\Gamma\left(\frac{d}{2}-x\right)}{\Gamma(x)} .
$$


This relation can also be represented diagrammatically as

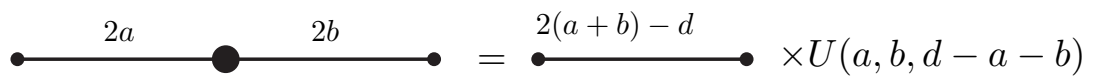

Propagator merging relations with fermions are given by

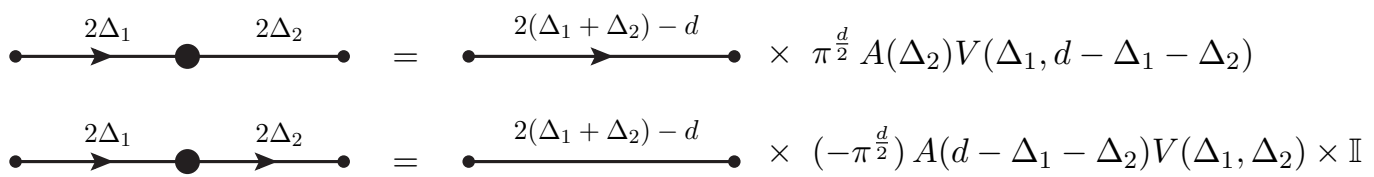

Here we defined

$$
V\left(\Delta_{1}, \Delta_{2}\right)=\frac{\Gamma\left(\frac{d}{2}-\Delta_{1}+\frac{1}{2}\right)}{\Gamma\left(\Delta_{1}+\frac{1}{2}\right)} \frac{\Gamma\left(\frac{d}{2}-\Delta_{2}+\frac{1}{2}\right)}{\Gamma\left(\Delta_{2}+\frac{1}{2}\right)} .
$$

Uniqueness relation, valid for $a_{1}+a_{2}+a_{3}=d$, has the form

$$
\int d^{d} x \frac{1}{\left|x_{1}-x\right|^{2 a_{1}}\left|x_{2}-x\right|^{2 a_{2}}\left|x_{3}-x\right|^{2 a_{3}}}=\frac{U\left(a_{1}, a_{2}, a_{3}\right)}{\left|x_{12}\right|^{d-2 a_{3}}\left|x_{13}\right|^{d-2 a_{2}}\left|x_{23}\right|^{d-2 a_{1}}},
$$

and can be represented graphically as
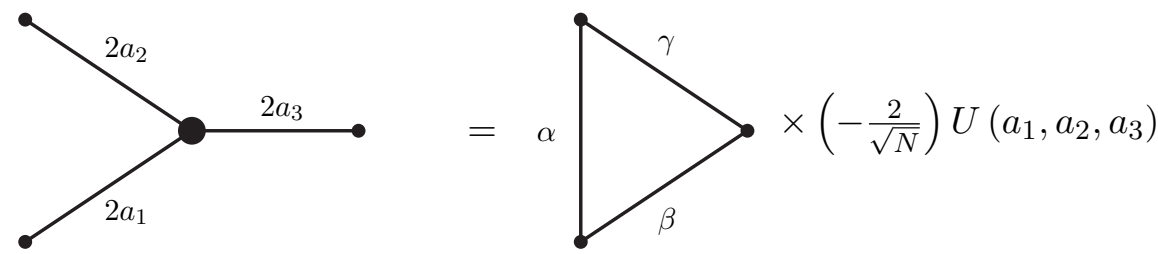

Here we have defined $\alpha=d-2 a_{3}, \beta=d-2 a_{2}, \gamma=d-2 a_{1}$.

In the case of a Yukawa vertex, the uniqueness relation becomes (for $\Delta_{1}+\Delta_{2}+\Delta_{3}=d$ )

$$
\int d^{d} x_{4} \frac{\gamma_{\mu} x_{41}^{\mu} \gamma_{\nu} x_{24}^{\nu}}{\left|x_{14}\right|^{2 \Delta_{1}+1}\left|x_{24}\right|^{2 \Delta_{2}+1}\left|x_{34}\right|^{2 \Delta_{3}}}=\frac{\pi^{\frac{d}{2}} A\left(\Delta_{3}\right) V\left(\Delta_{1}, \Delta_{2}\right) \gamma_{\mu} x_{31}^{\mu} \gamma_{\nu} x_{23}^{\nu}}{\left|x_{12}\right|^{d-2 \Delta_{3}}\left|x_{13}\right|^{d-2 \Delta_{2}+1}\left|x_{23}\right|^{d-2 \Delta_{1}+1}},
$$

or diagrammatically,
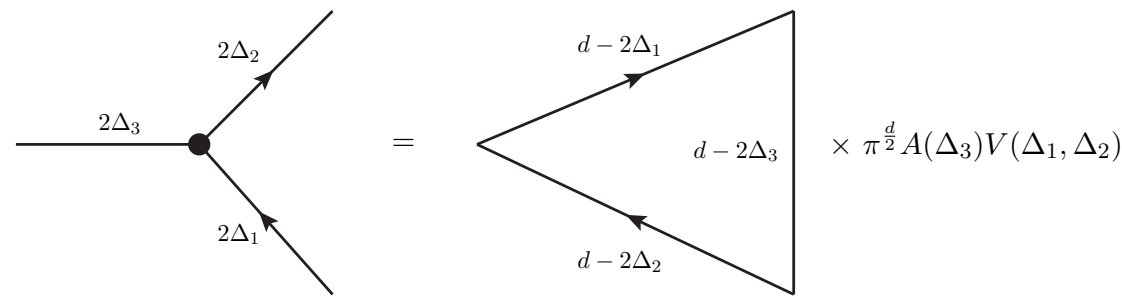

Open Access. This article is distributed under the terms of the Creative Commons Attribution License (CC-BY 4.0), which permits any use, distribution and reproduction in any medium, provided the original author(s) and source are credited. 


\section{References}

[1] D.J. Gross and A. Neveu, Dynamical symmetry breaking in asymptotically free field theories, Phys. Rev. D 10 (1974) 3235 [inSPIRE].

[2] J. Zinn-Justin, Four fermion interaction near four-dimensions, Nucl. Phys. B 367 (1991) 105 [inSPIRE].

[3] A.M. Polyakov, Conformal symmetry of critical fluctuations, JETP Lett. 12 (1970) 381 [Pisma Zh. Eksp. Teor. Fiz. 12 (1970) 538] [InSPIRE].

[4] K.G. Wilson and M.E. Fisher, Critical exponents in 3.99 dimensions, Phys. Rev. Lett. 28 (1972) 240 [InSPIRE].

[5] S.S. Gubser and I.R. Klebanov, A Universal result on central charges in the presence of double trace deformations, Nucl. Phys. B 656 (2003) 23 [hep-th/0212138] [INSPIRE].

[6] G. Parisi, The theory of nonrenormalizable interactions. 1. The large $N$ expansion, Nucl. Phys. B 100 (1975) 368 [inSPIRE].

[7] L. Fei, S. Giombi and I.R. Klebanov, Critical $O(N)$ models in $6-\epsilon$ dimensions, Phys. Rev. D 90 (2014) 025018 [arXiv: 1404.1094] [INSPIRE].

[8] L. Fei, S. Giombi, I.R. Klebanov and G. Tarnopolsky, Three loop analysis of the critical $O(N)$ models in $6-\varepsilon$ dimensions, Phys. Rev. D 91 (2015) 045011 [arXiv:1411.1099] [InSPIRE].

[9] J.A. Gracey, Four loop renormalization of $\phi^{3}$ theory in six dimensions, Phys. Rev. D 92 (2015) 025012 [arXiv:1506.03357] [InSPIRE].

[10] F.J. Dyson, Existence of a phase transition in a one-dimensional Ising ferromagnet, Commun. Math. Phys. 12 (1969) 91 [INSPIRE].

[11] M.E. Fisher, S.-k. Ma and B.G. Nickel, Critical exponents for long-range interactions, Phys. Rev. Lett. 29 (1972) 917 [InSPIRE].

[12] J.M. Kosterlitz, Phase transitions in long-range ferromagnetic chains, Phys. Rev. Lett. 37 (1976) 1577 [INSPIRE].

[13] M. Aizenman and R. Fernandez, Critical exponents for long-range interactions, Lett. Math. Phys. 16 (1988) 39.

[14] S.S. Gubser, C. Jepsen, S. Parikh and B. Trundy, $O(N)$ and $O(N)$ and $O(N)$, JHEP 11 (2017) 107 [arXiv:1703.04202] [INSPIRE].

[15] S. Giombi and H. Khanchandani, $O(N)$ models with boundary interactions and their long range generalizations, JHEP 08 (2020) 010 [arXiv: 1912.08169] [INSPIRE].

[16] N. Chai, M. Goykhman and R. Sinha, Long-range vector models at large N, JHEP 09 (2021) 194 [arXiv:2107.08052] [INSPIRE].

[17] J. Sak, Recursion relations and fixed points for ferromagnets with long-range interactions, Phys. Rev. B 8 (1973) 281.

[18] J. Sak, Low-temperature renormalization group for ferromagnets with long-range interactions, Phys. Rev. B 15 (1977) 4344.

[19] C. Behan, L. Rastelli, S. Rychkov and B. Zan, Long-range critical exponents near the short-range crossover, Phys. Rev. Lett. 118 (2017) 241601 [arXiv:1703.03430] [INSPIRE]. 
[20] C. Behan, L. Rastelli, S. Rychkov and B. Zan, A scaling theory for the long-range to short-range crossover and an infrared duality, J. Phys. A 50 (2017) 354002 [arXiv: 1703.05325] [INSPIRE].

[21] M.F. Paulos, S. Rychkov, B.C. van Rees and B. Zan, Conformal invariance in the long-range Ising model, Nucl. Phys. B 902 (2016) 246 [arXiv: 1509.00008] [InSPIRE].

[22] C. Behan, Bootstrapping the long-range Ising model in three dimensions, J. Phys. A 52 (2019) 075401 [arXiv:1810.07199] [inSPIRE].

[23] D.C. Brydges, P.K. Mitter and B. Scoppola, Critical $\left(\Phi^{4}\right)(3, \epsilon)$, Commun. Math. Phys. 240 (2003) 281 [hep-th/0206040] [INSPIRE].

[24] A. Abdesselam, A complete renormalization group trajectory between two fixed points, Commun. Math. Phys. 276 (2007) 727 [math-ph/0610018] [INSPIRE].

[25] E. Brezin, G. Parisi and F. Ricci-Tersengh, The crossover region between long-range and short-range interactions for the critical exponents, J. Stat. Phys. 157 (2014) 855.

[26] G. Slade, Critical exponents for long-range $O(n)$ models below the upper critical dimension, Commun. Math. Phys. 358 (2018) 343 [arXiv:1611.06169] [INSPIRE].

[27] D. Benedetti, R. Gurau, S. Harribey and K. Suzuki, Long-range multi-scalar models at three loops, J. Phys. A 53 (2020) 445008 [arXiv:2007.04603] [InSPIRE].

[28] S. Chakraborty and M. Goykhman, Critical long-range vector model in the UV, JHEP 10 (2021) 151 [arXiv:2108.10084] [INSPIRE].

[29] A. Giuliani, V. Mastropietro and S. Rychkov, Gentle introduction to rigorous Renormalization Group: a worked fermionic example, JHEP 01 (2021) 026 [arXiv: 2008.04361] [INSPIRE].

[30] J.A. Gracey, Calculation of exponent $\eta$ to $O\left(1 / N^{2}\right)$ in the $O(N)$ Gross-Neveu model, Int. J. Mod. Phys. A 6 (1991) 395 [Erratum ibid. 6 (1991) 2755] [INSPIRE].

[31] M. Goykhman, V. Rosenhaus and M. Smolkin, The background field method and critical vector models, JHEP 02 (2021) 074 [arXiv: 2009.13137] [INSPIRE].

[32] A.N. Manashov and M. Strohmaier, Correction exponents in the Gross-Neveu-Yukawa model at $1 / N^{2}$, Eur. Phys. J. C $\mathbf{7 8}$ (2018) 454 [arXiv:1711.02493] [InSPIRE].

[33] L. Di Pietro, E. Lauria and P. Niro, $3 d$ large $N$ vector models at the boundary, SciPost Phys. 11 (2021) 050 [arXiv: 2012.07733] [InSPIRE].

[34] M. Moshe and J. Zinn-Justin, Quantum field theory in the large $N$ limit: a review, Phys. Rept. 385 (2003) 69 [hep-th/0306133] [INSPIRE].

[35] A.N. Manashov and E.D. Skvortsov, Higher-spin currents in the Gross-Neveu model at 1/N², JHEP 01 (2017) 132 [arXiv: 1610.06938] [INSPIRE].

[36] A.N. Vasiliev and M.Y. Nalimov, Analog of dimensional regularization for calculation of the renormalization group functions in the $1 / n$ expansion for arbitrary dimension of Space, Theor. Math. Phys. 55 (1983) 423 [Teor. Mat. Fiz. 55 (1983) 163] [InSPIRE].

[37] A.C. Petkou, $C(T)$ and $C(J)$ up to next-to-leading order in $1 / N$ in the conformally invariant $O(N)$ vector model for $2<d<4$, Phys. Lett. B 359 (1995) 101 [hep-th/9506116] [INSPIRE].

[38] A. Petkou, Conserved currents, consistency relations and operator product expansions in the conformally invariant $O(N)$ vector model, Annals Phys. 249 (1996) 180 [hep-th/9410093] [INSPIRE]. 
[39] M. Goykhman and M. Smolkin, Vector model in various dimensions, Phys. Rev. D 102 (2020) 025003 [arXiv: 1911.08298] [InSPIRE].

[40] N. Chai, M. Goykhman and R. Sinha, Conformal correlators in the critical $O(N)$ vector model, arXiv:2103.10168 [INSPIRE].

[41] A.N. Vasiliev, Y.M. Pismak and Y.R. Khonkonen, Simple method of calculating the critical indices in the 1/N expansion, Theor. Math. Phys. 46 (1981) 104 [Teor. Mat. Fiz. 46 (1981) 157] [INSPIRE].

[42] A.N. Vasiliev, Y.M. Pismak and Y.R. Khonkonen, $1 / N$ expansion: calculation of the exponents $\eta$ and $\nu$ in the order $1 / N^{2}$ for arbitrary number of dimensions, Theor. Math. Phys. 47 (1981) 465 [Teor. Mat. Fiz. 47 (1981) 291] [InSPIRE].

[43] V.V. Belokurov and N.I. Usyukina, An algorithm for calculating massless Feynman diagrams, Acta Phys. Polon. B 14 (1983) 747 [InSPIRE].

[44] M. Ciuchini, S.E. Derkachov, J.A. Gracey and A.N. Manashov, Computation of quark mass anomalous dimension at $O\left(1 / N^{2}(f)\right)$ in quantum chromodynamics, Nucl. Phys. B 579 (2000) 56 [hep-ph/9912221] [INSPIRE].

[45] N. Chai, A. Dymarsky and M. Smolkin, Model of persistent breaking of discrete symmetry, Phys. Rev. Lett. 128 (2022) 011601 [arXiv:2106.09723] [INSPIRE].

[46] N. Chai, S. Chaudhuri, C. Choi, Z. Komargodski, E. Rabinovici and M. Smolkin, Thermal order in conformal theories, Phys. Rev. D 102 (2020) 065014 [arXiv: 2005. 03676] [InSPIRE].

[47] N. Chai, E. Rabinovici, R. Sinha and M. Smolkin, The bi-conical vector model at $1 / N$, JHEP 05 (2021) 192 [arXiv:2011.06003] [INSPIRE].

[48] M. D'Eramo, G. Parisi and L. Peliti, Theoretical predictions for critical exponents at the $\lambda$ point of Bose liquids, Lett. Nuovo Cim. 2 (1971) 878 [INSPIRE].

[49] K. Symanzik, On calculations in conformal invariant field theories, Lett. Nuovo Cim. 3 (1972) 734 [INSPIRE].

[50] M. Preti, STR: a Mathematica package for the method of uniqueness, Int. J. Mod. Phys. C 31 (2020) 2050146 [arXiv:1811.04935] [INSPIRE]. 